\title{
Influence of Nutrition, Food and Diet-Related Interventions in the Workplace: A Meta-Analysis with Meta-Regression
}

\author{
Liliana Melián-Fleitas ${ }^{1,2}$, Álvaro Franco-Pérez ${ }^{3}$, Pablo Caballero ${ }^{4} \mathbb{D}$, María Sanz-Lorente ${ }^{5,6}$, \\ Carmina Wanden-Berghe ${ }^{7}$ and Javier Sanz-Valero ${ }^{5,8, *(\mathbb{D})}$
}

1 Nutrition Department, University of Granada, 18012 Granada, Spain; lilianamelian@hotmail.es

2 Geriatric Service, Insular Hospital, Health Services Management of the Health Area of Lanzarote, 35500 Arrecife, Spain

3 Playa Blanca Health Center, Health Services Management of the Health Area of Lanzarote, 35580 Playa Blanca, Spain; amoisesfp@hotmail.com

4 Department of Community Nursing, Preventive Medicine and Public Health and History of Science, University of Alicante, San Vicente del Raspeig, 03690 Alicante, Spain; pablo.caballero@ua.es

5 Department of Public Health \& History of Science, University Miguel Hernandez, 03550 Alicante, Spain; msanzlor@gmail.com

6 Center of Public Health, Consellería of Universal Health and Public Health, 46940 Manises, Spain

7 Health and Biomedical Research Institute of Alicante, University General Hospital, 03010 Alicante, Spain; carminaw@telefonica.net

8 National School of Occupational Medicine, Carlos III Health Institute, 28029 Madrid, Spain

* Correspondence: fj.sanz@isciii.es

check for

updates

Citation: Melián-Fleitas, L.; Franco-Pérez, Á.; Caballero, P.; Sanz-Lorente, M.; Wanden-Berghe, C. Sanz-Valero, J. Influence of Nutrition, Food and Diet-Related Interventions in the Workplace: A Meta-Analysis with Meta-Regression. Nutrients 2021, 13, 3945. https://doi.org/10.3390/ nu13113945

Academic Editor: Rosa Casas

Received: 18 September 2021

Accepted: 2 November 2021

Published: 4 November 2021

Publisher's Note: MDPI stays neutral with regard to jurisdictional claims in published maps and institutional affiliations.

Copyright: (c) 2021 by the authors. Licensee MDPI, Basel, Switzerland. This article is an open access article distributed under the terms and conditions of the Creative Commons Attribution (CC BY) license (https:/ / creativecommons.org/licenses/by/ $4.0 /)$.

\begin{abstract}
Objective: To review the scientific literature on the influence of verified nutrition, food and diet interventions on occupational health. Method: This study involved a critical analysis of articles retrieved from MEDLINE (via PubMed), Embase, Cochrane Library, PsycINFO, Scopus, Web of Science, Latin American and Caribbean Health Sciences Literature (LILACS) and Medicina en Español (MEDES) using the descriptors "Diet, Food, and Nutrition" and "Occupational Health" and applying the filters "Clinical Trial", "Humans" and "Adult: 19+ years"; the search was conducted on 29 May 2021. Results: A total of 401 references were retrieved from the bibliographic databases, with an additional 16 identified through a secondary search; among the studies retrieved, 34 clinical trials were selected after applying the inclusion and exclusion criteria. The interventions were grouped into seven categories: (1) dietary interventions associated with exercise or educational programs; (2) individual environmental interventions or other educational actions; (3) educational interventions oriented toward lifestyle, dietetics, physical activity and stress management; (4) economic incentives; (5) multicomponent interventions (combination of mindfulness, e-coaching and the addition of fruits and vegetables); or dietary interventions (facilitating greater food supply in cafeterias); or interventions focused on physical exercise. Conclusions: Given that most people spend a large part of their time in the workplace and, therefore, eat at least one of their daily meals there, well-planned interventions-preferably including several strategies-have been demonstrated, in general, as useful for combating overweight and obesity. From the meta-regression study, it was observed that the interventions give better results in people who presented high Body Mass Index (BMI) values (obesity). In contrast, intervention 2 (interventions related to workplace environment) would not give the expected results (it would increase the BMI).
\end{abstract}

Keywords: diet; food; nutrition; occupational health; working conditions; workplace; obesity; overweight; occupational health policy

\section{Introduction}

The importance of good health, physical activity and adequate nutrition is frequently discussed. However, there are many occasions in which we do not realize that health and work go hand-in-hand, influencing each other. In this relationship, it must be taken into 
account that a large number of people eat at least one of their daily meals in the workplace, which makes food very important in working life.

A proper diet together with adequate hydration has the potential to influence many aspects of work. However, well-designed nutritional interventions as measures to improve the health and performance of workers are scarce [1]. Importantly, nutrition is an essential part of economic development because it influences the health and productivity of workers [2,3].

Community health focuses on the influence of adequate food/nutrition (diet) on occupational health and how to address dietary limitations (malnutrition) and dietary excesses (obesity). In this sense, the National Institute for Occupational Safety and Health $(\mathrm{NIOSH})$, which is part of the US Centers for Disease Control and Prevention, aims to raise awareness among employers and empower workers to create safe and healthy workplaces. NIOSH encourages "Total Worker Health", a strategy that integrates occupational safety and health protection to prevent worker injuries and illnesses and improve their health and well-being, with access to healthy and affordable food being an important topic [4].

The worldwide prevalence of overweight and obesity has tripled since the mid-1970s. Data for 2016 show that more than 1.9 billion adults were overweight, of whom more than 650 million were obese [5]. This trend is based on overeating, sedentary behaviors, unhealthy lifestyles, insufficient levels of physical activity, poor diet (highly caloric and processed foods), as well as a higher proportion of sedentary occupations [6,7].

Obesity significantly increases the risk of developing metabolic disorders, hypertension, coronary heart disease, stroke, dyslipidemia, type 2 diabetes, sleep apnea, acute respiratory distress syndrome and several types of cancer. In addition, it is associated with an increase in mortality and a low quality of life [8].

Additionally, this morbidity is also related to indirect costs, defined as losses due to reduced labor productivity. In fact, obesity and related diseases have been associated with an increased risk of workplace absenteeism (refers to the time taken off work due to sick leave, disability, injuries, or other reasons), presenteeism (refers to situations where people continue to work while unwell and not functioning to their full capacity) and permanent loss of work, which includes pensions for disability and premature death, generating massive costs for governments, society and employers [7,9]. In fact, productivity losses due to sick leave and presenteeism are even greater than the direct costs of medical treatment (an average of 2.30 USD in lost productivity for every dollar in medical expenses) [10].

This evidence urges governments, scientific organizations and companies to implement occupational safety and health measures, policies and global strategies that focus on organizational, behavioral and environmental factors related to work and that directly influence the overall health of workers and companies, paying special attention to nutrition. Consequently, companies and institutions have the responsibility of ensuring that the foods available in the workplace are nutritionally adequate or making unhealthy options unavailable.

Among the current trends focused on nutrition and occupational health, the creation of a new concept, nutra-ergonomics, stands out. Nutra-ergonomics is defined as the interface between workers, their work environment and their performance in relation to their nutritional status. Nutrition is an integral part of a safe and productive workplace that encompasses physical and mental health as well as the long-term well-being of workers [1].

From this global perspective, health and well-being programs in the workplace are presented as the best tools to address this growing problem; these programs comprise a set of coordinated strategies (including programs, policies, benefits, environmental support and links to the surrounding community) that are implemented in the workplace, designed to improve the health and safety of all employees [11], and there are studies that support the effectiveness of these programs in improving employee health and productivity $[1,11,12]$.

In addition, there is a general consensus that the combination of multicomponent interventions (focused on lifestyle management that includes stress management, physical 
activity, nutrition, and controlling smoking and alcohol consumption) is more effective than programs that focus on a single intervention (only exercise, for example) $[13,14]$.

The objective of this review was to review the scientific literature on the influence of verified nutrition, food and diet interventions on occupational health.

\section{Materials and Methods}

\subsection{Design}

This was a cross-sectional descriptive study and critical analysis of studies retrieved through a systematic review. The structure of this review followed the Preferred Reporting Items for Systematic Reviews and Meta-Analyses (PRISMA) guidelines, and the methodological framework proposed by Arksey \& O'Malley [15] for scoping studies.

\subsection{Source of Data Collection}

The data were obtained from direct consultation and access, via the internet, to the following bibliographic databases in the field of health sciences: MEDLINE (via PubMed), Embase, Cochrane Library, PsycINFO, Scopus, Web of Science, and Latin American \& Caribbean Health Sciences Literature (LILACS) and Medicina en Español (MEDES).

\subsection{Unit of Analysis}

We analyzed articles published and retrieved from the indicated bibliographic databases.

\subsection{Information Processing}

Search terms were selected using the Thesaurus of Health Sciences Descriptors (DeCS) developed by the Latin American and Caribbean Center on Health Sciences Information (BIREME) and equivalent terms established by the US National Library of Medicine, Medical Subject Headings (MeSH).

Based on the hierarchy of both thesaurus and their indexing files, the following search equations were considered adequate:

- Equation (1): Occupational Health

"Occupational Health" [Mesh] OR "Occupational Health" [Title/Abstract] OR "Industrial Hygiene" [Title/Abstract] OR "Industrial Health" [Title/Abstract] OR "Occupational Safety" [Title/Abstract] OR "Employee Health" [Title/Abstract] OR "Occupational exposure" [Mesh] OR "Occupational exposure" [Title/Abstract] OR "Occupational stress" [Mesh] OR "Occupational stress" [Title/Abstract] OR "Occupational diseases" [Mesh] OR "Occupational diseases" [Title/Abstract] OR "Occupational hazards" [Title/Abstract] OR "Occupational medicine" [Mesh] OR "Occupational medicine" [Title/Abstract] OR "Occupational health safety" [Title/Abstract] OR "Occupational Health Services" [Title/Abstract] OR "Occupational Health Services" [Mesh] OR "National Institute for Occupational Safety and Health (U.S.)" [Mesh] OR "Occupational stressors" [Title/Abstract] OR "Occupational stressor" [Title/Abstract] OR "Occupational Factors "[Title/Abstract] OR "Workplace" [Mesh] OR "Workplace" [Title/Abstract] OR "Workplace Health" [Title/Abstract] OR "Workplace safety" [Title/Abstract] OR "Safety climate" [Title/Abstract] OR "Total worker health" [Title/Abstract] OR "Working Environment" [Title/Abstract] OR "Job Satisfaction" [Mesh] OR "Job Satisfaction" [Title/Abstract] OR "Job Stress" [Title/Abstract] OR "Job security" [Title/Abstract] OR "Psychosocial working conditions" [Title/Abstract] OR "Employee Health" [Title/Abstract].

- Equation (2): Diet, Food, and Nutrition

"Diet, Food, and Nutrition" [Mesh] OR "Nutritional Status" [Mesh] OR "Nutritional Status" [Title/Abstract] OR "Nutrition Therapy" [Mesh] OR "Nutrition Therapy" [Title/Abstract] OR "Nutrition Assessment" [Mesh] OR "Nutrition Assessment" [Title/Abstract] OR "Nutrition Surveys" [Mesh] OR "Nutrition Surveys" [Title/Abstract] OR "Diet" [Mesh] OR "Diet" [Title/Abstract] OR "Healthy Diet" [Mesh] OR "Healthy Diet" [Title/Abstract] OR "Healthy Eating" [Title/Abstract] OR "Energy Intake" [Mesh] 
OR "Energy Intake" [Title/Abstract] OR "Meals" [Mesh] OR "Meals" [Title/Abstract] OR "Meal Time" [Title/Abstract] OR "Dinner Time" [Title/Abstract] OR "Breakfast" [Mesh] OR "Breakfast" [Title/Abstract] OR "Breakfast Time "[Title/Abstract] OR "Morning Meal" [Title/Abstract] OR "Food Services" [Mesh] OR "Food Services" [Title/Abstract] OR "Eating Practices" [Title/Abstract] OR "Dietary practices" [Title/Abstract] OR "Unhealthy food options" [Title/Abstract] OR "Eat and drink" [Title/Abstract] OR "Meal breaks" [Title/Abstract] OR "Dietary habits" [Title/Abstract] OR "Eating behavior" [Title/Abstract] OR "Meal timing" [Title/Abstract] OR "Eating at night" [Title/Abstract] OR "Body weight" [Title/Abstract] OR "BMI" [Title/Abstract] OR "Shiftwork" [Title/Abstract] OR "Work Hygiene" [Title/Abstract] OR "Healthy Lifestyle" [Mesh] OR "Feeding Behavior" [Mesh] OR "Feeding Behavior" [Title/Abstract] OR "Feeding Behaviors" [Title/Abstract] OR "Eating Behaviors" [Title/Abstract] OR "Feeding Patterns" [Title/Abstract] OR "Feeding Pattern" [Title/Abstract] OR "Food Habits" [Title/Abstract] OR "Food Habit" [Title/Abstract] OR "Eating Habits" [Title/Abstract] OR "Eating Habit" [Title/Abstract] OR "Diet Habits" [Title/Abstract] OR “Diet Habit" [Title/Abstract].

The final search equation was developed for use in MEDLINE via PubMed through the Boolean union of the 2 proposed equations (Equation (1) AND Equation (2)) using the filters Clinical Trial, Humans and Adult: 19+ years.

This strategy was subsequently adapted to the characteristics of each of the other databases consulted, performing the search from the first available date in each of the selected databases until 29 May 2021. Additionally, a complementary search strategy was performed to reduce the possibility of publication bias by manually searching the reference lists of the clinical trials that were selected for the review. Likewise, experts in the subject under study were contacted to determine the possible existence of gray literature (materials and research produced by organizations outside traditional commercial or academic publications that are disseminated through other distribution channels).

\subsection{Final Selection of Articles}

For the review and critical analysis, articles that met the following criteria were chosen:

- Inclusion: met the objectives of the search; clinical trial; published in a peer-reviewed journal and written in English, Spanish or Portuguese.

- Exclusion: full text could not be found; no relationship between the intervention and the outcome under study (causality criterion), and included a nonadult population (under 18 years of age).

The selection of relevant articles was performed by two authors of the present review (L.M-F. and A.F-P.). To validate the inclusion of the articles, the assessment of the agreement between the authors (kappa index $=$ KI) had to be greater than 0.60 [16]. Provided that this condition was met, possible disagreements were resolved by consensus among all authors of the review.

\subsection{Completeness of Reporting, Level of Evidence and Grade of Recommendation}

The adequacy of the selected articles was assessed using the CONSORT (Consolidated Standards of Reporting Trials) guidelines for reporting clinical trials [17]. This checklist contains 25 essential elements (items) that should be described in this type of study. One point was assigned for each item present (if not applicable, the item was not scored). When an item comprised several points, each was evaluated independently, giving the same weight to each point, and then the points for the item were averaged to obtain a final result, therefore, in no case was it possible to score more than 1 point per item.

To determine the level of evidence and its degree of recommendation, the recommendations of the Scottish Intercollegiate Guidelines Network Grading Review Group (SIGN) [18] were used. 


\subsection{Data Extraction}

Data correction was performed by inputting the data into duplicate tables, thus allowing the detection of deviations and their correction through consultation of the original document.

The elimination of duplicate records (present in more than one database) was executed using the multiplatform program ZOTERO (bibliographic reference manager developed by the Center for History and New Media of George Mason University).

To determine the actuality of the studies, the Burton-Kebler half-period (BK) and the Price index (PI) were calculated.

The articles were grouped based on the variables under study to systematize and facilitate the understanding of the results, considering the following data: first author, year of publication, population studied, pathology of the population, country where the study was developed, period of the study, type of intervention performed, and main results influenced by the effect of the intervention.

\subsection{Data Analysis}

Data related to information retrieval are presented as frequencies and percentages.

To determine the BK, the median age was calculated based on the time range analyzed, and the PI was calculated as the percentage of articles 5 years old or newer.

The measure of agreement to determine the relevance of the selected articles was performed using the KI. The agreement between authors was considered valid when the KI value was greater than $60 \%$ (good or very good agreement).

The scores of the CONSORT questionnaire were analyzed using the median, maximum and minimum. The evolution of this score, in relation to the years of publication, was obtained by Pearson's correlation analysis.

\subsection{Meta-Analysis and Meta-Regression}

To find out the effects of the interventions on workers' BMI, we analyzed the effect size using a meta-analysis of the studies included in the systematic review. The estimated model was the random-effects model. The results of the effect size and its $95 \%$ confidence interval were presented in the Forest plot, together with the percentage of heterogeneity, the Tau value for the contrast and the corresponding heterogeneity test.

Publication bias occurs when only favorable results are published, and it is suspected that studies with non-significant results failed to be published. The absence of such studies may overestimate the results. In this study, the Funnel plot has been used. In the Funnel plot, the effect measure of each study is plotted on the $x$-axis and a measure of precision, such as the standard error on the $y$-axis. A meta-analysis without publication bias would show a point cloud in the shape of an inverted funnel. Based on this assumption, we performed the non-parametric trim-and-fill analysis proposed by Duval and Tweedie [19], adjusting for the number of missing studies and re-estimating the results by including these missing studies. Another approach to estimating the number of missing studies was proposed by Copas et al. [20], which we have also used.

Meta-regression was used to determine whether intervention type or baseline BMI status would influence heterogeneity and effect sizes. Bivariate and multivariate models were applied. Baseline BMI status was divided into three groups, normal weight, overweight and obese, and five interventions were studied.

The results of the articles selected from the systematic review are shown by their authors in three different ways: the results before and after the interventions in terms of mean and standard deviation, the difference between before and after the interventions in terms of mean and $95 \% \mathrm{CI}$ and finally, the difference between before and after the interventions in terms of mean and standard deviation. In order to unify the criteria, the last option was used in the meta-analysis. Therefore, for the first situation, the difference of means and the weighted standard deviation were calculated for the first case. For the 
second case, the estimated standard error was obtained from the width of the $95 \% \mathrm{CI}$ and the sample size.

All calculations were performed in the R programming environment using the packages meta version 4.10-0 and metas version 0.4-0 [21].

\subsection{Ethical Aspects}

All data were obtained from published articles. Therefore, and in accordance with Spanish Law 14/2007, approval by an ethics committee was not necessary for the use of secondary data.

\section{Results}

A total of 401 articles were retrieved: 121 (30.17\%) in MEDLINE (via PubMed), 47 $(11.72 \%)$ in Embase, 62 (15.46\%) in Cochrane Library, 82 (20.45\%) in Scopus, 33 (8.23\%) in Web of Science, $50(12.47 \%)$ in PsycINFO and six $(1.502 \%)$ in MEDES. No documents were found in the LILACS bibliographic database. Consultation of the bibliographic lists of selected articles allowed the identification of another 16 studies.

After filtering the 75 repeated records and applying the inclusion and exclusion criteria (Figure 1), 34 clinical trials [22-55] were selected for review and critical analysis (see Table 1).
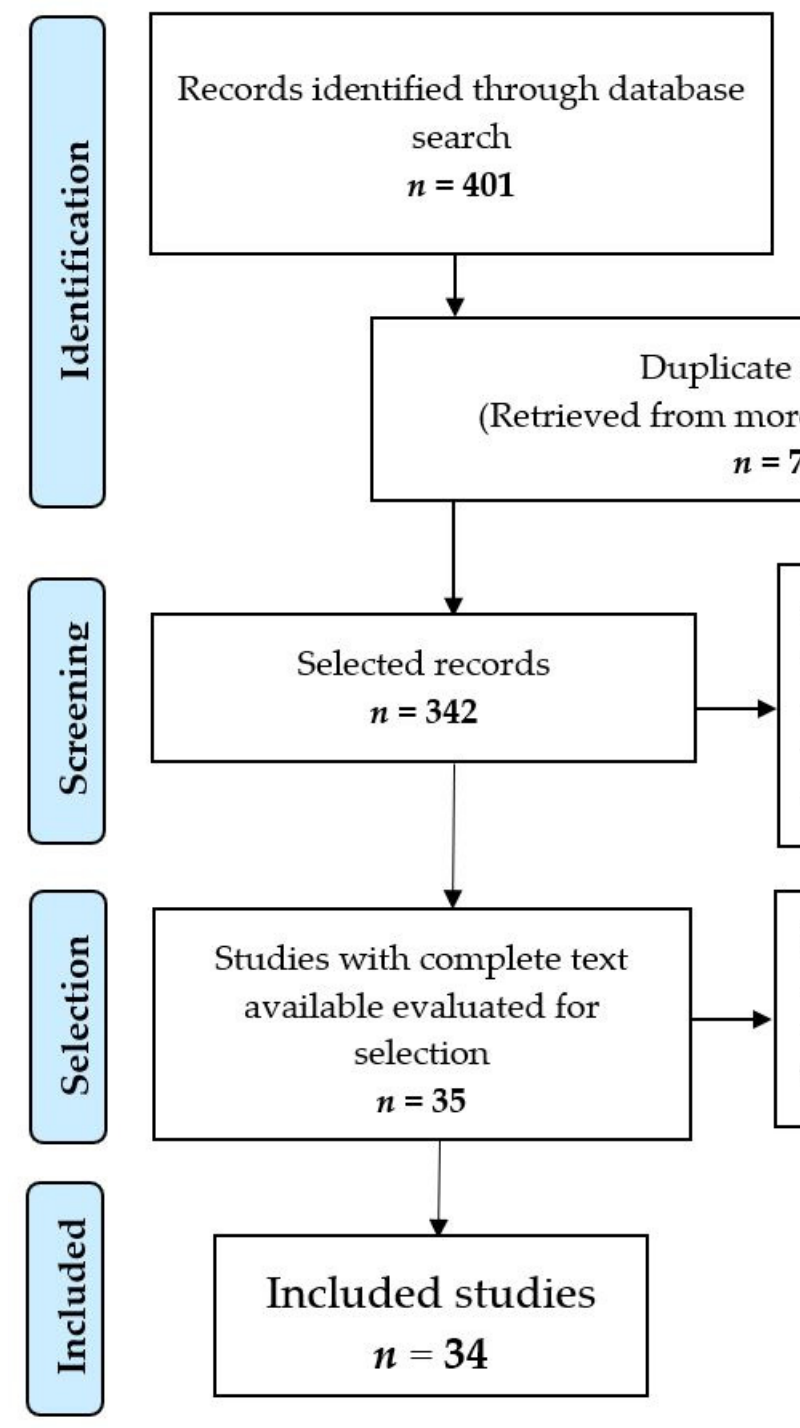

Additional studies identified from other sources (manual search and contribution by experts) $n=16$

Excluded records: $\boldsymbol{n}=\mathbf{3 0 7}$

- Not meeting the purpose of the review: 155

- Not clinical trial/design/baseline data: 137

- Non-working population: 13

- Language different from those established: 2

Excluded studies: $\boldsymbol{n}=\mathbf{1}$

- Underage working population: 0

- There was no causal relationship between the intervention and the outcome: 1

Figure 1. Selection procedure of the studies. 
Table 1. Summary of the studies reviewed.

\begin{tabular}{|c|c|c|c|c|c|c|}
\hline Author, Year & Population Studied & Pathology & Country & $\begin{array}{l}\text { Intervention } \\
\text { Period }\end{array}$ & $\begin{array}{c}\text { Type of } \\
\text { Intervention }\end{array}$ & Observed Outcome \\
\hline $\begin{array}{c}\text { Thorndike et al., } \\
2021 \text { [22] }\end{array}$ & $\begin{array}{l}\mathrm{N}=602 \text { Massachusetts } \\
\text { General Hospital } \\
\text { employees. } \\
\text { IG: } n=299 \\
\mathrm{M} / \mathrm{F}=69 / 230 \\
\text { Age Mean } \pm \mathrm{SD}= \\
43.5 \pm 12 \\
\mathrm{BMI} \pm \mathrm{SD}=28.6 \pm 6.6 \\
\mathrm{CG}: n=303 \\
\mathrm{M} / \mathrm{F}=55 / 248 \\
\text { Age Mean } \pm \mathrm{SD}= \\
43.8 \pm 12.5 \\
\mathrm{BMI} \pm \mathrm{SD}=28.0 \pm 6.5\end{array}$ & $\begin{array}{l}\text { Overweight } \\
\text { and obesity }\end{array}$ & USA & 12 months & $\begin{array}{l}\text { IG: Participants } \\
\text { received two emails } \\
\text { per week with } \\
\text { feedback on } \\
\text { previous cafeteria } \\
\text { purchases and } \\
\text { personalized health } \\
\text { and lifestyle tips } \\
\text { and one letter per } \\
\text { month with peer } \\
\text { comparisons and } \\
\text { financial incentives } \\
\text { for healthier } \\
\text { purchases. Emails } \\
\text { and letters were } \\
\text { automatically } \\
\text { generated using } \\
\text { survey, health, and } \\
\text { cafeteria data. } \\
\text { CG: Participants } \\
\text { received one letter } \\
\text { per month with } \\
\text { general healthy } \\
\text { lifestyle } \\
\text { information. }\end{array}$ & $\begin{array}{l}\text { There were no } \\
\text { between-group } \\
\text { differences in } \\
\text { weight change at } \\
12 / 24 \text { months. The } \\
\text { IG increased } \\
\text { green-labeled } \\
\text { purchases and } \\
\text { decreased } \\
\text { red-labeled and } \\
\text { calories purchased } \\
\text { compared with CG } \\
\text { ( } p<0.001) \text { at } \\
\text { 12/24 months. The } \\
\text { findings suggest } \\
\text { that an automated } \\
\text { behavioral } \\
\text { intervention using } \\
\text { workplace cafeteria } \\
\text { data improved } \\
\text { employees' food } \\
\text { choices but did not } \\
\text { prevent weight gain. }\end{array}$ \\
\hline $\begin{array}{l}\text { Röhling et al., } \\
2020 \text { [23] }\end{array}$ & $\begin{array}{l}\mathrm{N}=30 \text { Düsseldorf } \\
\text { Catholic Hospital } \\
\text { employees } \\
\text { IG: Starting } \\
\text { intervention (SI): } \\
n=15 \\
\mathrm{M} / \mathrm{F}=3 / 12 \\
\text { Age Mean } \pm \mathrm{SD}= \\
44 \pm 9 \\
\mathrm{BMI} \pm \mathrm{SD}=35.1 \pm 6.9 \\
\mathrm{CG}: \text { Waiting list }(\mathrm{WL}) \\
n=15 \\
\mathrm{M} / \mathrm{F}=2 / 13 \\
\text { Age Mean } \pm \mathrm{SD}= \\
49 \pm 7 \\
\mathrm{BMI} \pm \mathrm{SD}=32.8 \pm 6.1\end{array}$ & $\begin{array}{l}\text { Overweight } \\
\text { and obesity }\end{array}$ & Germany & 12 weeks & $\begin{array}{l}\text { All participants } \\
\text { were equipped with } \\
\text { telemetric devices } \\
\text { (scales and } \\
\text { pedometers). } \\
\text { IG: Immediately } \\
\text { started with } \\
\text { SAMMAS } \\
\text { intervention } \\
\text { (group-based } \\
\text { seminars, } \\
\text { low-carbohydrate } \\
\text { nutrition including } \\
\text { formula diet, } \\
\text { continuous glucose } \\
\text { monitoring, } \\
\text { telemetric } \\
\text { monitoring, and } \\
\text { telemedical } \\
\text { coaching) with } \\
\text { weekly contacts. } \\
\text { CG: Continued their } \\
\text { habitual lifestyle. At } \\
12 \text { weeks, they } \\
\text { started the same } \\
\text { SAMMAS } \\
\text { intervention. }\end{array}$ & $\begin{array}{l}\text { SI-group } \\
\text { significantly } \\
\text { reduced } \\
\text { weight ( } p<0.001) \\
\text { and improved in } \\
\text { BMI, WC, fat mass, } \\
\text { and all variables of } \\
\text { eating behavior (all } \\
p<0.05) \text { compared } \\
\text { to the WL-group } \\
\text { after } 12 \text { weeks of } \\
\text { intervention. The } \\
\text { Low-Insulin- } \\
\text { Method included in } \\
\text { the } \\
\text { multi-component, } \\
\text { occupational } \\
\text { healthcare program } \\
\text { SAMMAS could be } \\
\text { an effective and } \\
\text { promising new } \\
\text { approach for the } \\
\text { reduction in body } \\
\text { weight and } \\
\text { long-term weight } \\
\text { loss maintenance in } \\
\text { people with } \\
\text { overweight and } \\
\text { obesity. }\end{array}$ \\
\hline
\end{tabular}


Table 1. Cont

\begin{tabular}{|c|c|c|c|c|c|c|}
\hline Author, Year & Population Studied & Pathology & Country & $\begin{array}{l}\text { Intervention } \\
\text { Period }\end{array}$ & $\begin{array}{c}\text { Type of } \\
\text { Intervention }\end{array}$ & Observed Outcome \\
\hline $\begin{array}{c}\text { Iturriaga et al., } \\
2019 \text { [24] }\end{array}$ & $\begin{array}{l}\mathrm{N}=63 \text { (workers } \\
\text { not described) } \\
\mathrm{IG}: n=34 \\
\mathrm{M} / \mathrm{F}=0 / 34 \\
\text { Age Mean } \pm \mathrm{SD}= \\
42.53 \pm 5.34 \\
\mathrm{BMI} \pm \mathrm{SD}= \\
23.78 \pm 3.52 \\
\mathrm{CG}: n=29 \\
\mathrm{M} / \mathrm{F}=0 / 29 \\
\text { Age Mean } \pm \mathrm{SD}= \\
45.01 \pm 4.93 \\
\mathrm{BMI} \pm \mathrm{SD}= \\
25.64 \pm 5.12\end{array}$ & $\begin{array}{l}\text { Overweight } \\
\text { and obesity }\end{array}$ & Spain & 12 weeks & $\begin{array}{l}\text { IG: A } \\
\text { moderate-intensity } \\
\text { aerobic physical } \\
\text { exercise program } \\
\text { consisting of two } \\
\text { different activities, } \\
\text { Zumba or Aqua } \\
\text { fitness. Three } \\
\text { sessions per week } \\
\text { ( } 36 \text { sessions in total) } \\
\text { of duration } 45 \text { min } \\
\text { per session. }\end{array}$ & $\begin{array}{l}\text { Beneficial effects on } \\
\text { body composition of } \\
\text { a short-term } \\
\text { workplace aerobic } \\
\text { exercise program } \\
\text { (12 weeks) were } \\
\text { observed in terms of } \\
\text { reduced BMI } \\
(p=0.004) \text {, fat } \\
\text { indexes, and fat } \\
\text { mass }(p=0.001) \text { in } \\
\text { the lower limbs } \\
\text { compared to } \\
\text { controls. }\end{array}$ \\
\hline $\begin{array}{l}\text { Day et al., } \\
2019 \text { [25] }\end{array}$ & $\begin{array}{l}\mathrm{N}=421 \text { firefighters } \\
\mathrm{IG}: n=217 \\
\mathrm{M} / \mathrm{F}=168 / 49 \\
\text { Age Mean } \pm \mathrm{SD}= \\
37.3 \pm 12.7 \\
\mathrm{BMI}(\%), \\
(\mathrm{BMI}<25)=27.4 \\
(\mathrm{BMI} 25-29.9)=32.1 \\
(\mathrm{BMI} \geq 30)=40.5 \\
\mathrm{CG}: n=178 \\
\mathrm{M} / \mathrm{F}=149 / 29 \\
\text { Age Mean } \pm \mathrm{SD}= \\
36.9 \pm 12.6 \\
\mathrm{BMI}(\%), \\
(\mathrm{BMI}<25)=16.3 \\
(\mathrm{BMI} 25-29.9)=32.6 \\
(\mathrm{BMI} \geq 30)=51.1\end{array}$ & $\begin{array}{l}\text { Overweight } \\
\text { and obesity }\end{array}$ & USA & 6 months & $\begin{array}{l}\text { IG: They were } \\
\text { provided TF20 (First } \\
\text { Twenty } \\
\text { Intervention) } \\
\text { web-based program } \\
\text { and information } \\
\text { about enrollment } \\
\text { and use. The TF20 } \\
\text { includes modules on } \\
\text { physical activity, } \\
\text { nutrition and } \\
\text { behavioral health to } \\
\text { provide } 24 \text { weeks of } \\
\text { evidence-based } \\
\text { material. With } \\
\text { weekly goals, } \\
\text { messages vía emails, } \\
\text { tasks, resources and } \\
\text { tracking tools. } \\
\text { CG: Usual wellness } \\
\text { practices. They } \\
\text { received the same } \\
\text { introduction, } \\
\text { program overview, } \\
\text { follow-up and } \\
\text { evaluation as the IG. }\end{array}$ & $\begin{array}{l}\text { All models } \\
\text { indicated an average } \\
\text { weight gain for the } \\
\text { control group and } \\
\text { weight loss for the } \\
\text { treatment group. } \\
\text { The treatment effect } \\
\text { in the measured } \\
\text { weight of all } \\
\text { participants and } \\
\text { those overweight } \\
\text { and obese } \\
\text { approached } \\
\text { statistical } \\
\text { significance, } p=0.08 \\
\text { and } p=0.07, \\
\text { respectively, despite } \\
\text { the relatively small } \\
\text { samples. TF20 } \\
\text { supports firefighters' } \\
\text { weight loss. }\end{array}$ \\
\hline $\begin{array}{c}\text { Kempf et al., } \\
2019 \text { [26] }\end{array}$ & $\begin{array}{l}\mathrm{N}=104 \text { Boehringer } \\
\text { Ingelheim workers } \\
\text { IG Telemedical } \\
\text { coaching: } \\
n=34 \\
\mathrm{M} / \mathrm{F}=29 / 5 \\
\text { Age Mean } \pm \mathrm{SD}= \\
51 \pm 6 \\
\mathrm{BMI} \pm \mathrm{SD}=32 \pm 7 \\
\mathrm{CG} 1: n=34 \\
\mathrm{M} / \mathrm{F}=25 / 5 \\
\text { Age Mean } \pm \mathrm{SD}= \\
48 \pm 5 \\
\mathrm{BMI} \pm \mathrm{SD}=30 \pm 4\end{array}$ & Overweight & Germany & 12 months & $\begin{array}{l}\text { TMC and CG1 were } \\
\text { equipped with tele } \\
\text { monitoring devices } \\
\text { (scales and } \\
\text { pedometers) at } \\
\text { baseline and CG2 } \\
\text { after } 6 \text { months. All } \\
\text { participants were } \\
\text { instructed to } \\
\text { monitor their body } \\
\text { weight and physical } \\
\text { activity. }\end{array}$ & $\begin{array}{l}\text { All groups reduced } \\
\text { weight after } \\
12 \text { months }(p<0.01) \\
\text { and sustained it } \\
\text { during follow-up } \\
(p<0.01) \text {. All } \\
\text { groups reduced } \\
\text { BMI, systolic and } \\
\text { diastolic blood } \\
\text { pressure and } \\
\text { improved eating } \\
\text { behavior. }\end{array}$ \\
\hline
\end{tabular}


Table 1. Cont

\begin{tabular}{|c|c|c|c|c|c|c|}
\hline \multirow[t]{2}{*}{ Author, Year } & Population Studied & Pathology & Country & $\begin{array}{l}\text { Intervention } \\
\text { Period }\end{array}$ & $\begin{array}{c}\text { Type of } \\
\text { Intervention }\end{array}$ & Observed Outcome \\
\hline & $\begin{array}{l}\text { CG2: } n=36 \\
\mathrm{M} / \mathrm{F}=30 / 6 \\
\text { Age Mean } \pm \mathrm{SD}= \\
51 \pm 5 \\
\mathrm{BMI} \pm \mathrm{SD}=31 \pm 4\end{array}$ & & & & $\begin{array}{l}\text { IG: (TMC): Was } \\
\text { coached with } \\
\text { weekly care calls in } \\
\text { months } 3-6 \text { and } \\
\text { monthly calls from } \\
\text { months } 7 \text { to } 12 \text {. } \\
\text { CG1: Received no } \\
\text { further support. } \\
\text { CG2: Had a short } \\
\text { coaching phase in } \\
\text { months 6-9. }\end{array}$ & $\begin{array}{l}\text { TMC and/or tele } \\
\text { monitoring support } \\
\text { long-term weight } \\
\text { reduction in } \\
\text { overweight } \\
\text { employees. The } \\
\text { combination of both } \\
\text { interventions points } \\
\text { towards an } \\
\text { additional effect } \\
\text { (not supported by } \\
\text { the intention to treat } \\
\text { analysis). }\end{array}$ \\
\hline $\begin{array}{l}\text { Tene et al., } \\
2018 \text { [27] }\end{array}$ & $\begin{array}{l}\mathrm{N}=277 \text { research center } \\
\text { workers } \\
\text { G1 (Low-Fat Diet): } n= \\
139 \\
\mathrm{M} / \mathrm{F}: 122 / 17 \\
\text { Age Mean } \pm \mathrm{SD}=48.4 \\
\pm 9.2 \\
\mathrm{BMI} \pm \mathrm{SD}=30.8 \pm 3.7 \\
\mathrm{G} 2 \\
\text { (Mediterranean/Low- } \\
\text { Carbohydrate Diet): } \\
n=138 \\
\mathrm{M} / \mathrm{F}: 124 / 14 \\
\text { Age Mean } \pm \text { SD }= \\
47.5 \pm 9.3 \\
\text { BMI } \pm \text { SD = 30.9 } \pm 3.9 \\
\text { At } 6 \text { months of DI } \\
\text { randomized groups } \\
\text { with physical activity } \\
\text { for the last } 12 \text { months } \\
\text { of intervention: } \\
\text { G1: LF PA+ } \\
/ \text { MED-LC } \text { PA+ } \\
\text { G2 LF PA- } \\
/ \text { MED-LC PA- }\end{array}$ & $\begin{array}{l}\text { Abdominal } \\
\text { obesity or } \\
\text { dyslipi- } \\
\text { demia }\end{array}$ & Israel & 18 months & $\begin{array}{l}\text { Dietary intervention } \\
\text { (DI): } \\
1500 \text { kcal/day for } \\
\text { women and } 1800 \text { for } \\
\text { men (both diets), } \\
\text { included weekly } \\
\text { nutritional sessions. } \\
\text { - } \\
\text { Mediterranean/low- } \\
\text { carbohydrate diet: } \\
\text { Provided } 28 \text { gr } \\
\text { walnuts/day. } \\
\text {-Low Fat diet: limit } \\
\text { total fat intake to } \\
30 \% \text { of calories, up } \\
\text { to } 10 \% \text { of saturated } \\
\text { fat, and no more } \\
\text { than } 300 \text { mg of } \\
\text { cholesterol per day, } \\
\text { and to increase } \\
\text { dietary fibers. } \\
\text { Physical activity } \\
\text { (PA) intervention: } \\
\text { Free } 12-\text { month gym } \\
\text { membership, and } \\
\text { monthly } 60-\text { min } \\
\text { educational } \\
\text { workshops in the } \\
\text { workplace. }\end{array}$ & $\begin{array}{l}\text { At } 6 \text { months } \\
\text { pancreatic-fat } \\
\text { significantly } \\
\text { decreased } \\
(p=0.002) \text {, similarly } \\
\text { between diets } \\
(p=0.736) \text { and after } \\
18 \text { months } \\
\text { ( } p=0.049) \text {. This } \\
\text { study shows } \\
\text { modulation of } \\
\text { pancreatic fat } \\
\text { through lifestyle } \\
\text { interventions, } \\
\text { mainly by } \\
\text { increasing dietary } \\
\text { fat proportion on } \\
\text { the account of the } \\
\text { relative } \\
\text { carbohydrate intake. } \\
\text { The efficacy is best } \\
\text { achieved when } \\
\text { accompanied by } \\
\text { moderate endurance } \\
\text { exercise. }\end{array}$ \\
\hline $\begin{array}{l}\text { Viester et al., } \\
2017 \text { [28] }\end{array}$ & $\begin{array}{l}\mathrm{N}=314 \text { Construction } \\
\text { Workers } \\
\text { IG: } n=162 \\
\mathrm{M} / \mathrm{F}: 162 / 0 \\
\text { Age Mean } \pm \mathrm{SD}= \\
46.3 \pm 9.9 \\
\mathrm{BMI} \pm \mathrm{SD}=27.3 \pm 3.5 \\
\text { CG: } n=152 \\
\text { M/F: } 152 / 0\end{array}$ & $\begin{array}{l}\text { Overweight } \\
\text { and muscu- } \\
\text { loskeletal } \\
\text { disorders }\end{array}$ & Netherland & s 6 months & $\begin{array}{l}\text { IG: They received } \\
\text { individual coaching } \\
\text { sessions, tailored } \\
\text { information, and } \\
\text { materials to improve } \\
\text { lifestyle behavior } \\
\text { (Physical activity } \\
\text { and dietary } \\
\text { behavior). } \\
\text { CG: Received } \\
\text { usual care. }\end{array}$ & $\begin{array}{l}\text { Positive changes } \\
\text { showed in vigorous } \\
\text { physical activity and } \\
\text { intake of } \\
\text { sugar-sweetened } \\
\text { beverages compared } \\
\text { to controls, as well } \\
\text { as effects on body } \\
\text { weight }(p=0.010) \text {, } \\
\text { BMI }(p=0.010) \text {, and } \\
\text { waist circumference } \\
(p=0.032) \text { at } \\
6 \text { months. }\end{array}$ \\
\hline
\end{tabular}


Table 1. Cont.

\begin{tabular}{|c|c|c|c|c|c|c|}
\hline Author, Year & Population Studied & Pathology & Country & $\begin{array}{l}\text { Intervention } \\
\text { Period }\end{array}$ & $\begin{array}{c}\text { Type of } \\
\text { Intervention }\end{array}$ & Observed Outcome \\
\hline & $\begin{array}{l}\text { Age Mean } \pm \mathrm{SD}= \\
47.0 \pm 9.5 \\
\mathrm{BMI} \pm \mathrm{SD}=27.4 \pm 3.9\end{array}$ & & & & & $\begin{array}{l}\text { Long-term effects } \\
\text { were still promising } \\
\text { but not statistically } \\
\text { significant. }\end{array}$ \\
\hline $\begin{array}{l}\text { Shrivastava et al., } \\
2017 \text { [29] }\end{array}$ & $\begin{array}{l}\mathrm{N}=267 \text { corporative } \\
\text { workers } \\
\text { IG: } n=156 \\
\mathrm{M} / \mathrm{F}: 137 / 19 \\
\text { Age Mean } \pm \mathrm{SD}= \\
35.8 \pm 7.6 \\
\mathrm{BMI} \pm \mathrm{SD}= \\
28.21 \pm 2.89 \\
\mathrm{CG}: n=111 \\
\mathrm{M} / \mathrm{F}: 92 / 19 \\
\text { Age Mean } \pm \mathrm{SD}= \\
39.0 \pm 8.7 \\
\mathrm{BMI} \pm \mathrm{SD}= \\
28.20 \pm 3.59\end{array}$ & Overweight & India & 6 months & $\begin{array}{l}\text { IG: A } \\
\text { multicomponent } \\
\text { intervention with } \\
\text { sessions on the } \\
\text { different topics } \\
\text { related to healthy } \\
\text { living, diet, stress } \\
\text { and physical activity. } \\
\text { And monitoring the } \\
\text { compliance of } \\
\text { lifestyle changes } \\
\text { with digital } \\
\text { resources. } \\
\text { CG: No intervention, } \\
\text { but were given } \\
\text { general health talk } \\
\text { twice in six months. }\end{array}$ & $\begin{array}{l}\text { Active intervention } \\
\text { was successful in } \\
\text { achieving of } \\
\text { reduction in weight, } \\
\text { excess subcutaneous } \\
\text { fat, and } \\
\text { cardiometabolic risk } \\
\text { factors after } \\
6 \text { months. } \\
\text { Statistically } \\
\text { significant changes } \\
\text { in IG vs. CG in } \\
\text { weight, BMI, waist } \\
\text { circumference, hip } \\
\text { circumference } \\
(p<0.001) \text {. }\end{array}$ \\
\hline $\begin{array}{l}\text { Gepner et al., } \\
2017 \text { [30] }\end{array}$ & $\begin{array}{l}\mathrm{N}=278 \text { research } \\
\text { center workers } \\
\text { G1 (Low-Fat Diet) } \\
n=139 \\
\mathrm{M} / \mathrm{F}: 122 / 17 \\
\text { Age Mean } \pm \mathrm{SD}= \\
48.4 \pm 9.2 \\
\mathrm{BMI} \pm \mathrm{SD}=30.8 \pm 3.7 \\
\mathrm{G} 2 \\
\text { (Mediterranean/Low- } \\
\text { Carbohydrate Diet): } \\
n=139 \\
\mathrm{M} / \mathrm{F}: 125 / 14 \\
\text { Age Mean } \pm \text { SD }= \\
47.4 \pm 9.3 \\
\text { BMI } \pm \text { SD = 30.9 } \pm 4.0 \\
\text { At } 6 \text { months of DI } \\
\text { randomized groups } \\
\text { with physical activity } \\
\text { for the last } 12 \text { months } \\
\text { of intervention: } \\
\text { G1: LF PA+ } \\
/ \text { MED-LC PA+ } \\
n=126 \\
\text { G2 LF PA- } \\
/ \text { MED-LC PA- } \\
n=130\end{array}$ & $\begin{array}{l}\text { Abdominal } \\
\text { obesity or } \\
\text { dyslipi- } \\
\text { demia }\end{array}$ & Israel & 18 months & $\begin{array}{l}\text { Dietary intervention } \\
\text { (DI): Monitored } \\
\text { provided lunch } \\
\text { (1500 kcal/day } \\
\text { women and } \\
1800 \text { men) and a } \\
\text { 90-min nutritional } \\
\text { session in the } \\
\text { workplace with } \\
\text { clinical dietitians } \\
\text { every week (first } \\
\text { month), and every } \\
\text { month thereafter. } \\
\text { - } \\
\text { Mediterranean/low- } \\
\text { carbohydrate diet: } \\
\text { Provided } 28 \text { g } \\
\text { walnuts / day. } \\
\text {-Low Fat diet: limit } \\
\text { total fat intake to } \\
30 \% \text { of calories, up } \\
\text { to } 10 \% \text { of saturated } \\
\text { fat, and no more } \\
\text { than } 300 \text { mg of } \\
\text { cholesterol per day, } \\
\text { and to increase } \\
\text { dietary fibers. } \\
\text { Physical Activity } \\
\text { Intervention: Free } \\
\text { supervised gym } \\
\text { membership for } \\
12 \text { months, three } \\
\text { sessions per week, } \\
\text { included monthly } \\
60-\text { min educational } \\
\text { workshops. }\end{array}$ & $\begin{array}{l}\text { Energy intake } \\
\text { decreased similarly } \\
\text { across diet groups } \\
\text { after } 6 \text { months } \\
(p=0.85) \text { and } \\
18 \text { months }(p=0.18) \text {, } \\
\text { and all were } \\
\text { significantly lower } \\
\text { compared with } \\
\text { baseline ( } p<0.001) . \\
\text { A Mediterranean } \\
\text { diet, rich in } \\
\text { unsaturated fats and } \\
\text { low in } \\
\text { carbohydrates, and } \\
\text { being physically } \\
\text { active can improve } \\
\text { cardiometabolic risk } \\
\text { markers through } \\
\text { changes in } \\
\text { visceral/ectopic fat } \\
\text { depots that are not } \\
\text { reflected by mild } \\
\text { body weight } \\
\text { changes alone. }\end{array}$ \\
\hline
\end{tabular}


Table 1. Cont.

\begin{tabular}{|c|c|c|c|c|c|c|}
\hline Author, Year & Population Studied & Pathology & Country & $\begin{array}{l}\text { Intervention } \\
\text { Period }\end{array}$ & $\begin{array}{c}\text { Type of } \\
\text { Intervention }\end{array}$ & Observed Outcome \\
\hline $\begin{array}{l}\text { Faghri et al., } \\
2017 \text { [31] }\end{array}$ & $\begin{array}{l}\mathrm{N}=99 \text { nursing-home } \\
\text { employees } \\
\mathrm{M} / \mathrm{F}: 9 / 88 \\
\text { Age Mean } \pm \mathrm{SD}= \\
46.98 \pm 11.36 \\
\mathrm{BMI} \pm \mathrm{SD}= \\
35.33 \pm 6.91 \\
\text { IG: Incentivized } \\
\text { participants (IP): } \\
n=51 \\
\text { CG: Non incentivized } \\
\text { participants (NIP): } \\
n=48\end{array}$ & $\begin{array}{c}\text { Overweight, } \\
\text { obesity and } \\
\text { diabetes }\end{array}$ & USA & 16 weeks & $\begin{array}{l}\text { IG: Financial } \\
\text { incentive-based } \\
\text { intervention. All } \\
\text { participants } \\
\text { received a } \\
\text { personalized } \\
\text { weight-loss } \\
\text { consultation based } \\
\text { on their reported } \\
\text { physical activity } \\
\text { habits and dietary } \\
\text { preferences. Each } \\
\text { participant received } \\
\text { an action plan based } \\
\text { on the National } \\
\text { Diabetes Prevention } \\
\text { Program (NDPP) } \\
\text { CG: No incentive. }\end{array}$ & $\begin{array}{l}\text { IP reduced more } \\
\text { weight ( } p=0.027) \\
\text { and BMI ( } p=0.043) \\
\text { than NIP at week } 16 . \\
\text { At week 28, IP lost } \\
\text { more weight than } \\
\text { NIP ( } p=0.053) \text {, and } \\
\text { reduced their BMI } \\
\text { more than NIP } \\
\text { ( } p=0.308) . \text { Eating } \\
\text { and exercise } \\
\text { self-efficacy were } \\
\text { significant } \\
\text { mediators between } \\
\text { health behaviors } \\
\text { and weight loss } \\
\text { ( } p<0.05) \text { Incentives } \\
\text { significantly } \\
\text { moderated the } \\
\text { effects of } \\
\text { self-efficacy } \\
\text { ( } p=0.00) \text { on weight } \\
\text { loss. Self-efficacy } \\
\text { and financial } \\
\text { incentives may } \\
\text { affect weight loss } \\
\text { and play a role in } \\
\text { weight-loss } \\
\text { interventions. }\end{array}$ \\
\hline
\end{tabular}

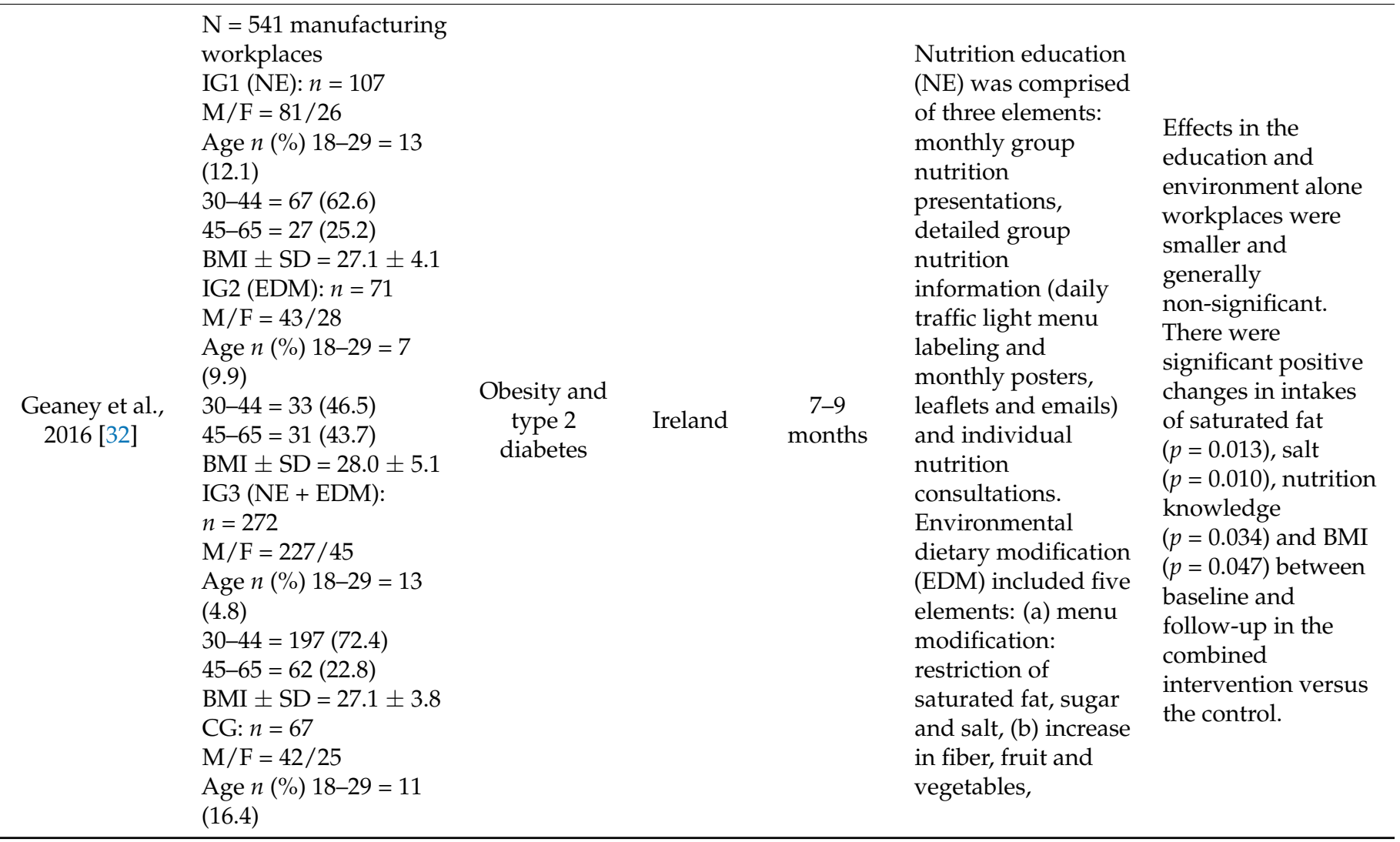


Table 1. Cont.

\begin{tabular}{|c|c|c|c|c|c|c|}
\hline \multirow[t]{2}{*}{ Author, Year } & Population Studied & Pathology & Country & $\begin{array}{l}\text { Intervention } \\
\text { Period }\end{array}$ & $\begin{array}{c}\text { Type of } \\
\text { Intervention }\end{array}$ & Observed Outcome \\
\hline & $\begin{array}{l}30-44=34(50.7) \\
45-65=22(32.8) \\
\mathrm{BMI} \pm \mathrm{SD}=27.6 \pm 4.2\end{array}$ & & & & $\begin{array}{l}\text { (c) price discounts } \\
\text { for whole fresh fruit, } \\
\text { (d) strategic } \\
\text { positioning of } \\
\text { healthier } \\
\text { alternatives and (e) } \\
\text { portion size control. }\end{array}$ & $\begin{array}{l}\text { Combining nutrition } \\
\text { education and } \\
\text { environmental } \\
\text { dietary modification } \\
\text { may be an effective } \\
\text { approach for } \\
\text { promoting a healthy } \\
\text { diet and weight loss } \\
\text { at work. }\end{array}$ \\
\hline $\begin{array}{l}\text { Solenhill et al., } \\
2016 \text { [33] }\end{array}$ & $\begin{array}{l}\mathrm{N}=981 \text { transportation } \\
\text { companies employees } \\
\mathrm{M} / \mathrm{F}: 655 / 326 \\
\text { Age Mean } \pm \mathrm{SD}= \\
44 \pm 10.2 \\
\mathrm{IG} 1 \text { (intervention Web): } \\
n=301 \\
\mathrm{BMI} \pm \mathrm{SD}=26.6 \pm 4.4 \\
\mathrm{IG} 2 \text { (intervention } \\
\text { Web }+ \text { telephone): } \\
n=324 \\
\mathrm{BMI} \pm \mathrm{SD}=26.1 \pm 4.0 \\
\mathrm{CG}: n=356 \\
\mathrm{BMI} \pm \mathrm{SD}=26.6 \pm 4.5\end{array}$ & $\begin{array}{l}\text { Obesity, } \\
\text { diabetes, } \\
\text { and cardio- } \\
\text { vascular } \\
\text { diseases }\end{array}$ & Sweden & 9 months & $\begin{array}{l}\text { IG1: They received } \\
\text { tailored Web-based } \\
\text { health feedback. } \\
\text { IG2: They received } \\
\text { tailored Web-based } \\
\text { health feedback + } \\
\text { additional optional } \\
\text { telephone health } \\
\text { coaching for those } \\
\text { participants who } \\
\text { were motivated to } \\
\text { change health } \\
\text { behaviors. } \\
\text { CG: No additional } \\
\text { intervention. }\end{array}$ & $\begin{array}{l}\text { Tailored Web-based } \\
\text { health feedback and } \\
\text { the offering of } \\
\text { optional telephone } \\
\text { coaching did not } \\
\text { have a positive } \\
\text { health effect on } \\
\text { employees in the } \\
\text { transport services. }\end{array}$ \\
\hline
\end{tabular}

\begin{tabular}{|c|c|c|c|c|c|c|}
\hline $\begin{array}{l}\text { Mitchell et al., } \\
2015 \text { [34] }\end{array}$ & $\begin{array}{l}\mathrm{N}=254 \text { Latino } \\
\text { farmworkers } \\
\mathrm{M} / \mathrm{F}: 71 / 183 \\
\mathrm{IG}: n=174 \\
\text { Age Mean } \pm \mathrm{SD}= \\
32.3 \pm 7.6 \\
\mathrm{BMI} \pm \mathrm{SD}=29.1 \pm 0.3 \\
\mathrm{CG}: n=80 \\
\text { Age Mean } \pm \mathrm{SD}= \\
32.5 \pm 7.9 \\
\mathrm{BMI} \pm \mathrm{SD}=27.7 \pm 0.4\end{array}$ & $\begin{array}{l}\text { Overweight, } \\
\text { obesity and } \\
\text { diabetes }\end{array}$ & USA & 10 weeks & $\begin{array}{l}\text { IG: } 10 \text { weekly } \\
\text { educational sessions } \\
\text { (health habits, } \\
\text { physical activity and } \\
\text { dietary behaviors) } \\
\text { led by promoters. } \\
\text { CG: No } \\
\text { intervention. }\end{array}$ & $\begin{array}{l}\text { Greater losses in } \\
\text { weight }(p=0.0002) \text {, } \\
\text { BMI }(p=0.0001), \\
\text { and waist } \\
\text { circumference } \\
\text { ( } p=0.001) \text { were } \\
\text { associated with } \\
\text { increasing } \\
\text { attendance at } \\
\text { intervention } \\
\text { sessions. Women } \\
\text { significantly } \\
\text { reduced weight } \\
\text { ( } p=0.001) \text { and BMI } \\
\text { ( } p=0.002) \\
\text { compared with } \\
\text { controls, except } \\
\text { blood glucose. The } \\
\text { successful pilot } \\
\text { workplace } \\
\text { intervention offers a } \\
\text { model to reach } \\
\text { otherwise } \\
\text { difficult-to-access } \\
\text { Latino farmworkers. }\end{array}$ \\
\hline $\begin{array}{l}\text { Fernández et al., } \\
\quad 2015 \text { [35] }\end{array}$ & $\begin{array}{l}\mathrm{N}=2614 \\
\text { manufacturing, } \\
\text { research, and } \\
\text { development company } \\
\text { employees } \\
\text { IG: } n=1547 \\
\text { M/F: } 1054 / 493\end{array}$ & $\begin{array}{l}\text { Overweight } \\
\text { and obesity }\end{array}$ & USA & 2 years & $\begin{array}{l}\text { Environmental } \\
\text { intervention in the } \\
\text { worksite. } \\
\text { Employees received } \\
\text { a small economic } \\
\text { incentive. }\end{array}$ & $\begin{array}{l}\text { BMI decreased } \\
\text { significantly at the } \\
\text { intervention } \\
\text { worksites after } \\
2 \text { years }(p=0.03) \text { and } \\
\text { non-significantly at } \\
\text { the control worksites }\end{array}$ \\
\hline
\end{tabular}


Table 1. Cont.

\begin{tabular}{|c|c|c|c|c|c|c|}
\hline Author, Year & Population Studied & Pathology & Country & $\begin{array}{l}\text { Intervention } \\
\text { Period }\end{array}$ & $\begin{array}{c}\text { Type of } \\
\text { Intervention }\end{array}$ & Observed Outcome \\
\hline & $\begin{array}{l}\text { Age Mean } \pm \mathrm{SD}= \\
47.7 \pm 7.47 \\
\mathrm{BMI} \pm \mathrm{SD}=28.6 \pm 5.50 \\
\mathrm{CG}: n=1067 \\
\mathrm{M} / \mathrm{F}: 594 / 474 \\
\text { Age Mean } \pm \mathrm{SD}= \\
47.4 \pm 7.84 \\
\mathrm{BMI} \pm \mathrm{SD}=28.6 \pm 5.55\end{array}$ & & & & $\begin{array}{l}\text { The intervention } \\
\text { promotes healthy } \\
\text { lifestyles through } \\
\text { portion control, } \\
\text { education, healthy } \\
\text { diets, and physical } \\
\text { activity. }\end{array}$ & $\begin{array}{l}(p=0.6) \text {. Worksite } \\
\text { environmental } \\
\text { interventions may } \\
\text { be promising } \\
\text { strategies for } \\
\text { addressing weight } \\
\text { control at the } \\
\text { population level. }\end{array}$ \\
\hline $\begin{array}{c}\text { Almeida et al., } \\
2015 \text { [36] }\end{array}$ & $\begin{array}{l}28 \text { worksites (workers } \\
\text { not described) } \\
\mathrm{N}=1790 \text { employees } \\
\text { IG INCENT: } n=789 \\
\mathrm{M} / \mathrm{F}(\% \pm \mathrm{SD})= \\
\text { 19.79/80.21 } \pm 10.84 \\
\text { Age Mean } \pm \mathrm{SD}= \\
45.68 \pm 3.30 \\
\mathrm{BMI} \pm \mathrm{SD}= \\
33.26 \pm 6.39 \\
\mathrm{IG} \mathrm{LMW}: n=1001 \\
\mathrm{M} / \mathrm{F}(\% \pm \mathrm{SD})= \\
32.57 / 67.43 \pm 25.02 \\
\text { Age Mean } \pm \mathrm{SD}= \\
48.24 \pm 2.78 \\
\mathrm{BMI} \pm \mathrm{SD}= \\
33.51 \pm 6.44\end{array}$ & $\begin{array}{l}\text { Overweight, } \\
\text { and obesity }\end{array}$ & USA & 12 months & $\begin{array}{l}\text { Two weight loss } \\
\text { interventions } \\
\text { targeted diet and } \\
\text { physical activity } \\
\text { behaviors: } \\
\text { IG INCENT: } \\
\text { Individually } \\
\text { targeted } \\
\text { Internet-based } \\
\text { intervention with } \\
\text { monetary incentives. } \\
\text { INCENT was } \\
\text { delivered via daily } \\
\text { e-mails over } 12 \\
\text { months. } \\
\text { IG Livin' My Weigh } \\
\text { (LMW): a } \\
\text { less-intensive } \\
\text { minimal } \\
\text { intervention that } \\
\text { included } \\
\text { newsletters and } \\
\text { onsite educational } \\
\text { sessions delivered } \\
\text { on a quarterly basis. } \\
\text { LMW was delivered } \\
\text { quarterly via both } \\
\text { newsletters and } \\
\text { onsite educational } \\
\text { sessions. }\end{array}$ & $\begin{array}{l}\text { Participants in the } \\
\text { INCENT group on } \\
\text { average lost } \\
2.27 \text { lbs }(p<0.001) \\
\text { and had a BMI } \\
\text { decrease of } \\
0.36 \mathrm{~kg} / \mathrm{m}^{2} \\
(p<0.001) \text { while } \\
\text { participants in LMW } \\
\text { group lost } 1.30 \mathrm{lbs} \\
(p<0.05) \text { and } \\
\text { decreased BMI by } \\
0.20 \mathrm{~kg} / \mathrm{m}^{2} \\
(p<0.05) . \text { However, } \\
\text { the differences } \\
\text { between INCENT } \\
\text { and LMW groups in } \\
\text { weight loss and BMI } \\
\text { reduction were not } \\
\text { significant. Both } \\
\text { approaches } \\
\text { investigated were } \\
\text { successful in } \\
\text { helping participants } \\
\text { lose small amounts } \\
\text { of weight and } \\
\text { decrease their BMI. }\end{array}$ \\
\hline $\begin{array}{l}\text { Østbye et al., } \\
2015 \text { [37] }\end{array}$ & $\begin{array}{l}\mathrm{N}=550 \text { Duke } \\
\text { University and Medical } \\
\text { Center employees } \\
\text { IG1 (WM+ behavioral): } \\
n=275 \\
\text { M/F: } 45 / 230 \\
\text { Age: } \\
<35=42 \\
35-50=133 \\
>50=100 \\
\text { BMI } \pm \text { SD = } \\
37.37 \pm 6.61 \\
\text { IG2 (WM educational): } \\
n=275 \\
\text { M/F: } 48 / 227\end{array}$ & Obesity & USA & 1 year & $\begin{array}{l}\text { Weight } \\
\text { Management [WM]: } \\
\text { Educational } \\
\text { program targeting } \\
\text { healthy lifestyle } \\
\text { changes for weight } \\
\text { loss (portion control, } \\
\text { education, healthy } \\
\text { diets, and physical } \\
\text { activity). } \\
\text { Weight Management } \\
\text { Plus [WM+]: } \\
\text { Intensive behavioral } \\
\text { intervention: (1) } \\
\text { monthly counseling } \\
\text { sessions, (2) } \\
\text { meetings with an } \\
\text { exercise physiologist }\end{array}$ & $\begin{array}{l}\text { There were no } \\
\text { clinically, or } \\
\text { statistically, } \\
\text { meaningful } \\
\text { differences between } \\
\text { groups but there } \\
\text { were modest } \\
\text { reductions in body } \\
\text { mass index and } \\
\text { positive, meaningful } \\
\text { changes in diet and } \\
\text { physical activity for } \\
\text { both groups. }\end{array}$ \\
\hline
\end{tabular}


Table 1. Cont.

\begin{tabular}{|c|c|c|c|c|c|c|}
\hline Author, Year & Population Studied & Pathology & Country & $\begin{array}{l}\text { Intervention } \\
\text { Period }\end{array}$ & $\begin{array}{c}\text { Type of } \\
\text { Intervention }\end{array}$ & Observed Outcome \\
\hline & $\begin{array}{l}\text { Age: } \\
<35=53 \\
35-50=134 \\
>50=88 \\
\mathrm{BMI} \pm \mathrm{SD}= \\
37.02 \pm 6.14 \\
\text { Used in the analysis } \\
\text { WM }(n=220) \\
\mathrm{WM}+(n=215)\end{array}$ & & & & $\begin{array}{l}\text { (3) quarterly } \\
\text { biometric feedback, } \\
\text { (4) targeted health } \\
\text { education materials, } \\
\text { and (5) information } \\
\text { and active linking } \\
\text { with various Duke } \\
\text { programs and } \\
\text { wellness resources, } \\
\text { (6) use of eHealth } \\
\text { trackers for diet and } \\
\text { weight. }\end{array}$ & \\
\hline $\begin{array}{l}\text { Van Berkel } \\
\text { et al., } 2014 \text { [38] }\end{array}$ & $\begin{array}{l}\mathrm{N}=257 \text { research } \\
\text { institutes employees } \\
\mathrm{IG}: n=129 \\
\mathrm{M} / \mathrm{F}: 47 / 82 \\
\text { Age Mean } \pm \mathrm{SD}= \\
46.0 \pm 9.4 \\
\mathrm{BMI} \pm \mathrm{SD}= \\
24.74 \pm 3.96 \\
\mathrm{CG}: n=128 \\
\mathrm{M} / \mathrm{F}: 37 / 91 \\
\text { Age Mean } \pm \mathrm{SD}= \\
45.1 \pm 9.6 \\
\mathrm{BMI} \pm \mathrm{SD}= \\
24.66 \pm 3.56\end{array}$ & $\begin{array}{l}\text { Overweight } \\
\text { and obesity }\end{array}$ & Netherlands & 6 months & $\begin{array}{l}\text { IG: } 8 \text { weeks of } \\
\text { in-company } \\
\text { mindfulness } \\
\text { training with } \\
\text { homework exercises, } \\
\text { followed by eight } \\
\text { sessions of } \\
\text { e-coaching (in their } \\
\text { free time). } \\
\text { Additionally, free } \\
\text { fruit and snack } \\
\text { vegetables were } \\
\text { provided for } \\
6 \text { months. } \\
\text { CG: They received } \\
\text { information on } \\
\text { existing lifestyle } \\
\text { behavior-related } \\
\text { facilities that were } \\
\text { already available at } \\
\text { the worksite. }\end{array}$ & $\begin{array}{l}\text { This study did not } \\
\text { show an effect of a } \\
\text { worksite } \\
\text { mindfulness-based } \\
\text { multi-component } \\
\text { intervention on } \\
\text { lifestyle behaviors } \\
\text { and behavioral } \\
\text { determinants after } 6 \\
\text { and } 12 \text { months. }\end{array}$ \\
\hline $\begin{array}{c}\text { Mishra et al., } \\
2013 \text { [39] }\end{array}$ & $\begin{array}{l}\mathrm{N}=291 \mathrm{GEICO} \\
\text { corporate offices } \\
\text { employees } \\
\mathrm{IG}: n=142 \\
\mathrm{M} / \mathrm{F}: 32 / 110 \\
\text { Age Mean } \pm \mathrm{SD}= \\
44.3 \pm 15.3 \\
\mathrm{BMI} \pm \mathrm{SE}=34.7 \pm 0.6 \\
\mathrm{CG}: n=149 \\
\mathrm{M} / \mathrm{F}: 18 / 131 \\
\text { Age Mean } \pm \mathrm{SD}= \\
46.1 \pm 13.6 \\
\mathrm{BMI} \pm \mathrm{SE}=35.3 \pm 0.7\end{array}$ & $\begin{array}{c}\text { Overweight, } \\
\text { obesity and } \\
\text { type } 2 \\
\text { diabetes }\end{array}$ & USA & 18 weeks & $\begin{array}{l}\text { IG: a low-fat vegan } \\
\text { diet, with weekly } \\
\text { group support and } \\
\text { work cafeteria } \\
\text { options available } \\
\text { plus a daily } \\
\text { supplement of } \\
\text { vitamin B12. } \\
\text { CG: No Intervention. } \\
\text { They were given } \$ 50 \\
\text { gift certificates for } \\
\text { completion of all } \\
\text { aspects of the study. }\end{array}$ & $\begin{array}{l}\text { An } 18 \text {-week dietary } \\
\text { intervention using a } \\
\text { low-fat plant-based } \\
\text { diet in a corporate } \\
\text { setting improves } \\
\text { body weight } \\
(p<0.001), \text { BMI } \\
(p<0.001) \text {, plasma } \\
\text { lipids }(p=0.001) \text {, } \\
\text { and, in individuals } \\
\text { with diabetes, } \\
\text { glycemic control } \\
(p=0.003) .\end{array}$ \\
\hline $\begin{array}{l}\text { Salinardi et al., } \\
\quad 2013 \text { [40] }\end{array}$ & $\begin{array}{l}\mathrm{N}=466 \text { Boston } \\
\text { companies employees } \\
\text { IG: } n=84 \\
\mathrm{M} / \mathrm{F}: 21 / 63 \\
\text { Age Mean } \pm \mathrm{SD}= \\
48.6 \pm 1.2\end{array}$ & $\begin{array}{l}\text { Overweight } \\
\text { and obesity }\end{array}$ & USA & 12 months & $\begin{array}{l}\text { IG: Intervention } \\
\text { combined } \\
\text { recommendations to } \\
\text { consume a } \\
\text { reduced-energy, } \\
\text { low-glycemic load, } \\
\text { high-fiber diet with } \\
\text { behavioral change } \\
\text { education. }\end{array}$ & $\begin{array}{l}\text { Worksites can be } \\
\text { successful locations } \\
\text { for the } \\
\text { implementation of } \\
\text { interventions that } \\
\text { cause substantial } \\
\text { mean weight loss } \\
(p<0.001)\end{array}$ \\
\hline
\end{tabular}


Table 1. Cont.

\begin{tabular}{|c|c|c|c|c|c|c|}
\hline Author, Year & Population Studied & Pathology & Country & $\begin{array}{l}\text { Intervention } \\
\text { Period }\end{array}$ & $\begin{array}{c}\text { Type of } \\
\text { Intervention }\end{array}$ & Observed Outcome \\
\hline & $\begin{array}{l}\mathrm{BMI} \pm \mathrm{SD}=33.3 \pm 0.7 \\
\mathrm{CG}: n=34 \\
\mathrm{M} / \mathrm{F}: 8 / 26 \\
\text { Age Mean } \pm \mathrm{SD}= \\
49.9 \pm 2.1 \\
\mathrm{BMI} \pm \mathrm{SD}=33.3 \pm 1.2\end{array}$ & & & & $\begin{array}{l}\text { Employees who } \\
\text { completed the } \\
\text { weight-loss program } \\
\text { were invited to } \\
\text { reenroll in the 6-mo } \\
\text { program (identical } \\
\text { to the original } \\
\text { except that the } \\
\text { groups met once per } \\
\text { month). } \\
\text { CG: Wait-listed } \\
\text { weight-loss } \\
\text { program. }\end{array}$ & $\begin{array}{l}\text { and improve } \\
\text { cardiometabolic risk } \\
\text { factors (total } \\
\text { cholesterol, glucose, } \\
\text { systolic blood } \\
\text { pressure, and } \\
\text { diastolic blood } \\
\text { pressure, } p \leq 0.02 \\
\text { for each). }\end{array}$ \\
\hline $\begin{array}{l}\text { Christensen et al., } \\
\quad 2012 \text { [41] }\end{array}$ & $\begin{array}{l}\mathrm{N}=98 \text { health care } \\
\text { workers } \\
\mathrm{M} / \mathrm{F}: 0 / 98 \\
\text { IG: } n=54 \\
\mathrm{M} / \mathrm{F}: 0 / 54 \\
\text { Age Mean } \pm \mathrm{SD}= \\
45.7 \pm 8.7 \\
\mathrm{BMI} \pm \mathrm{SD}=30.7 \pm 5.4 \\
\mathrm{CG}: n=44 \\
\mathrm{M} / \mathrm{F}: 0 / 44 \\
\text { Age Mean } \pm \mathrm{SD}= \\
46.0 \pm 8.6 \\
\text { BMI } \pm \mathrm{SD}=30.4 \pm 4.9\end{array}$ & $\begin{array}{l}\text { Overweight } \\
\text { and obesity }\end{array}$ & Denmark & 12 months & $\begin{array}{l}\text { IG: one-hour weekly } \\
\text { workplace } \\
\text { intervention } \\
\text { consisting of diet, } \\
\text { physical exercise } \\
\text { and cognitive- } \\
\text { behavioral training. } \\
\text { CG: monthly } \\
\text { two-hour oral } \\
\text { presentation during } \\
\text { working hours } \\
\text { about the Danish } \\
\text { Dietary } \\
\text { recommendations } \\
\text { and other } \\
\text { health-related } \\
\text { topics. }\end{array}$ & $\begin{array}{l}\text { The intervention } \\
\text { generated } \\
\text { substantial } \\
\text { reductions in body } \\
\text { weight }(p<0.001) \text {, } \\
\text { BMI }(p<0.001) \text { and } \\
\text { body fat percentage } \\
(p<0.001) \text {. The } \\
\text { positive results } \\
\text { support the } \\
\text { workplace as an } \\
\text { efficient arena for } \\
\text { weight loss among } \\
\text { overweight females. }\end{array}$ \\
\hline $\begin{array}{c}\text { Thorndike et al., } \\
2012 \text { [42] }\end{array}$ & $\begin{array}{l}\mathrm{N}=330 \text { Massachusetts } \\
\text { General Hospital } \\
\text { employees } \\
\text { IG: } n=174 \\
\text { M/F: } 17 / 157 \\
\text { Age Mean } \pm \mathrm{SD}= \\
44.2 \pm 11.8 \\
\mathrm{BMI} \pm \mathrm{SD}=28.0 \pm 5.8 \\
\mathrm{CG}: n=156 \\
\mathrm{M} / \mathrm{F}: 28 / 128 \\
\text { Age Mean } \pm \mathrm{SD}= \\
\text { 41.6 } \pm 13.6 \\
\mathrm{BMI} \pm \mathrm{SD}=27.5 \pm 5.9\end{array}$ & $\begin{array}{l}\text { Overweight } \\
\text { and obesity }\end{array}$ & USA & 10 weeks & $\begin{array}{l}\text { Ten-week exercise } \\
\text { and nutrition } \\
\text { program (IG and } \\
\text { CG) immediately } \\
\text { following by } \\
\text { 9-month } \\
\text { maintenance } \\
\text { intervention. } \\
\text { IG: Internet support } \\
\text { with a website for } \\
\text { goal-setting and } \\
\text { self-monitoring of } \\
\text { weight and exercise } \\
\text { plus minimal } \\
\text { personal support } \\
\text { (for } 9 \text { months). } \\
\text { CG: usual care (for } \\
9 \text { months). }\end{array}$ & $\begin{array}{l}\text { The initial program } \\
\text { resulted in moderate } \\
\text { weight loss and } \\
\text { improvements in } \\
\text { diet and exercise } \\
\text { behaviors at } 1 \text { year } \\
(p<0.001) \text { in both } \\
\text { groups, but no } \\
\text { difference in weight } \\
\text { loss between groups } \\
\text { The Internet-based } \\
\text { maintenance } \\
\text { program } \\
\text { immediately } \\
\text { following did not } \\
\text { improve these } \\
\text { outcomes. }\end{array}$ \\
\hline $\begin{array}{l}\text { Linde et al., } \\
2012 \text { [43] }\end{array}$ & $\begin{array}{l}\mathrm{N}=1672 \text { in six } \\
\text { worksites } \\
\text { IG: } n=723 \\
\text { M/F: } 273 / 450\end{array}$ & $\begin{array}{l}\text { Overweight } \\
\text { and obesity }\end{array}$ & USA & 2 years & $\begin{array}{l}\text { IG: A } \\
\text { four-component } \\
\text { environmental } \\
\text { intervention focused } \\
\text { on food availability } \\
\text { and price, }\end{array}$ & $\begin{array}{l}\text { BMI was not } \\
\text { significantly affected } \\
\text { by environmental } \\
\text { changes. }\end{array}$ \\
\hline
\end{tabular}


Table 1. Cont.

\begin{tabular}{|c|c|c|c|c|c|c|}
\hline Author, Year & Population Studied & Pathology & Country & $\begin{array}{l}\text { Intervention } \\
\text { Period }\end{array}$ & $\begin{array}{c}\text { Type of } \\
\text { Intervention }\end{array}$ & Observed Outcome \\
\hline & $\begin{array}{l}\text { Age }(\%),<30=18.3 \% \\
31-40=24.3 \% \\
41-50=31.1 \% \\
51-60=23.4 \% \\
>60=3.0 \% \\
\text { BMI } \pm \text { SD }=28.7 \pm 6.6 \\
\text { CG: } n=949 \\
\text { M/F: } 40.5 \% / 59.5 \% \\
\text { Age }(\%),<30=15.6 \% \\
31-40=26.0 \% \\
41-50=31.9 \% \\
51-60=22.8 \% \\
>60=3.7 \% \\
\text { BMI } \pm S D=28.3 \pm 6.1\end{array}$ & & & & $\begin{array}{l}\text { physical activity } \\
\text { promotion, scale } \\
\text { access, and media } \\
\text { enhancements to } \\
\text { promote a healthier } \\
\text { workforce and } \\
\text { improve weight } \\
\text { control. } \\
\text { CG: Following the } \\
\text { last round of data } \\
\text { collection, control } \\
\text { sites were offered a } \\
\text { DVD containing } \\
\text { intervention } \\
\text { materials and an } \\
\text { opportunity to ask } \\
\text { questions of } \\
\text { intervention staff as } \\
\text { needed. }\end{array}$ & $\begin{array}{l}\text { Mean weight and } \\
\text { BMI gain was higher } \\
\text { at intervention sites } \\
\text { relative to controls. } \\
\text { Results about } \\
\text { environmental } \\
\text { change at worksites } \\
\text { may be not } \\
\text { sufficient for } \\
\text { population weight } \\
\text { gain prevention. }\end{array}$ \\
\hline
\end{tabular}

$\begin{array}{cl} & \text { N }=102 \text { employees of a } \\ \text { company in Kanagawa } & \text { Prefecture } \\ & \text { IG: } n=49 \\ & \text { M/F: } 49 / 0 \\ & \text { Age Mean } \pm \text { SD }= \\ \text { Nanri et al., } & 53.7 \pm 6.1 \\ 2012[44] & \text { BMI } \pm \text { SD }=26.0 \pm 2.4 \\ & \text { CG: } n=53 \\ & \text { M/F: } 53 / 0 \\ \text { Age Mean } \pm S D= \\ \text { 52.8 } \pm 7.4 \\ \text { BMI } \pm \text { SD }=25.6 \pm 2.3\end{array}$

Metabolic syndrome (MS)

Japan 6 months
IG: received a six-month lifestyle modification program focused on exercise and diet behavior from a trained occupational health nurse at the baseline and at one and three months. CG: Standard health guidance by an occupational health nurse using a leaflet at the baseline.
The program did not lead to a greater decrease in the prevalence of metabolic syndrome. However, WC $(p=0.02)$, body weight $(p<0.001)$, $\operatorname{BMI}(p=0.001)$ and glycated hemoglobin $(p=0.005)$ were significantly decreased in the intervention group, as well as a significant reduction in sugar and sweetener intake $(p=0.002)$, in cereal intake $(p=0.002)$ and an increase in physical activity $(p<0.001)$.

IG: Multicomponent environmental intervention that

$\mathrm{N}=341$ manufacturing companies employees

$\mathrm{M} / \mathrm{F}(\%)=60 / 40$

Brehm et al., Age Mean $\pm \mathrm{SD}=$ 2011 [45]
$43.8 \pm 10.0$
$\mathrm{BMI} \pm \mathrm{SD}=29.0 \pm 5.5$
IG: $n=168$
CG: $n=173$

Obesity

USA

1 year
There were no intervention effects for outcome variables. Findings indicate that subtle environmental changes alone may not impact employees' weight and health. included employee advisory committees, point-of-decision prompts, walking paths, cafeteria/vending changes, and educational materials. 
Table 1. Cont.

\begin{tabular}{|c|c|c|c|c|c|c|}
\hline Author, Year & Population Studied & Pathology & Country & $\begin{array}{l}\text { Intervention } \\
\text { Period }\end{array}$ & $\begin{array}{c}\text { Type of } \\
\text { Intervention }\end{array}$ & Observed Outcome \\
\hline $\begin{array}{l}\text { Christensen et al., } \\
2011 \text { [46] }\end{array}$ & $\begin{array}{l}\mathrm{N}=144 \text { health care } \\
\text { workers } \\
\mathrm{IG}: n=54 \\
\mathrm{M} / \mathrm{F}: 0 / 54 \\
\text { Age Mean } \pm \mathrm{SD}= \\
45.7 \pm 8.7 \\
\mathrm{BMI} \pm \mathrm{SD}=30.5 \pm 5.4 \\
\mathrm{CG}: n=44 \\
\mathrm{M} / \mathrm{F}: 0 / 44 \\
\text { Age Mean } \pm \mathrm{SD}= \\
46.0 \pm 8.6 \\
\mathrm{BMI} \pm \mathrm{SD}=30.4 \pm 4.9\end{array}$ & $\begin{array}{l}\text { Overweight } \\
\text { and obesity }\end{array}$ & Denmark & 12 months & $\begin{array}{l}\text { IG: An individually } \\
\text { dietary plan with an } \\
\text { energy deficit of } \\
1200 \mathrm{kcal} / \text { day, } \\
\text { strengthening } \\
\text { exercises and } \\
\text { cognitive- } \\
\text { behavioral training } \\
\text { during working } \\
\text { hours } 1 \mathrm{~h} / \text { week. } \\
\text { Leisure time aerobic } \\
\text { fitness was planned } \\
\text { for } 2 \text { h/week. } \\
\text { CG: Monthly oral } \\
\text { presentations. }\end{array}$ & $\begin{array}{l}\text { The significantly } \\
\text { reduced body } \\
\text { weight, body fat, } \\
\text { waist circumference } \\
\text { and blood pressure } \\
\text { as well as increased } \\
\text { aerobic fitness in the } \\
\text { intervention group } \\
\text { ( } p \leq 0.001) \text { show the } \\
\text { great potential of } \\
\text { workplace health } \\
\text { promotion among } \\
\text { this high-risk } \\
\text { workgroup. }\end{array}$ \\
\hline $\begin{array}{l}\text { Barham et al., } \\
2011 \text { [47] }\end{array}$ & $\begin{array}{l}\mathrm{N}=45 \text { employees of } \\
\text { Onondaga County } \\
\text { Department of } \\
\text { Probation, Health and } \\
\text { Social Services } \\
\text { IG: } n=21 \\
\text { M/F: } 4 / 17 \\
\text { Age Mean } \pm \mathrm{SD}= \\
51.1 \pm 9.6 \\
\text { BMI } \pm \text { SD }=39.4 \pm 6.9 \\
\text { CG: } n=24 \\
\text { M/F: } 3 / 21 \\
\text { Age Mean } \pm S D= \\
51.2 \pm 6.4 \\
\text { BMI } \pm \text { SD }=36 \pm 6.9\end{array}$ & $\begin{array}{c}\text { Overweight, } \\
\text { obesity and } \\
\text { type } 2 \\
\text { diabetes }\end{array}$ & USA & 3 months & $\begin{array}{l}\text { IG: 3-month } \\
\text { program (12 } \\
\text { one-hour weekly } \\
\text { midday group } \\
\text { sessions) that } \\
\text { targeted healthy } \\
\text { diet, physical } \\
\text { activity, and stress } \\
\text { reduction, followed } \\
\text { by a monthly } \\
\text { maintenance } \\
\text { program with the } \\
\text { groups choosing } \\
\text { topics that they } \\
\text { considered of } \\
\text { greatest benefit. } \\
\text { CG: Wait list control } \\
\text { group. }\end{array}$ & $\begin{array}{l}\text { The IG lost } \\
\text { significant weight } \\
\text { compared to the } \\
\text { wait CG over the } \\
\text { first } 3 \text { months, with } \\
\text { a decrease in BMI } \\
(p<0.001) \text { and waist } \\
\text { circumference } \\
(p=0.004) \text {, an } \\
\text { increase in physical } \\
\text { activity ( } p=0.011) \\
\text { and lower dietary } \\
\text { fat intake ( } p=0.018) \text {. } \\
\text { A worksite } \\
\text { intervention } \\
\text { program can help } \\
\text { government } \\
\text { employees adopt } \\
\text { healthier lifestyles } \\
\text { and achieve modest } \\
\text { weight loss. }\end{array}$ \\
\hline $\begin{array}{l}\text { Ferdowsian et al., } \\
2010 \text { [48] }\end{array}$ & $\begin{array}{l}\mathrm{N}=113 \mathrm{GEICO} \\
\text { company employees } \\
\text { IG: } n=68 \\
\mathrm{M} / \mathrm{F}: 18 / 50 \\
\text { Age Mean } \pm \mathrm{SD}= \\
46 \pm 10 \\
\mathrm{BMI} \pm \mathrm{SD}=\text { Not } \\
\text { provided } \\
\mathrm{CG}: n=45 \\
\mathrm{M} / \mathrm{F}: 2 / 43 \\
\text { Age Mean } \pm \mathrm{SD}= \\
42 \pm 10 \\
\text { BMI } \pm \mathrm{SD}=\mathrm{Not} \\
\text { provided }\end{array}$ & $\begin{array}{l}\text { Overweight, } \\
\text { obesity and } \\
\text { diabetes } \\
\text { type } 2\end{array}$ & USA & 22 weeks & $\begin{array}{l}\text { IG: Follow a low-fat } \\
\text { vegan diet for } \\
22 \text { weeks, group } \\
\text { meetings, cooking } \\
\text { demonstrations. } \\
\text { Also provided with } \\
\text { practical tools and a } \\
\text { grocery store tour. } \\
\text { CG: They were } \\
\text { compensated with } \\
\text { gift certificates }(\$ 60) \\
\text { and informed that } \\
\text { the nutrition } \\
\text { program would be } \\
\text { provided upon } \\
\text { study completion. }\end{array}$ & $\begin{array}{l}\text { IG participants } \\
\text { experienced greater } \\
\text { weight changes } \\
\text { compared with CG } \\
(p<0.0001) \text {, as well } \\
\text { as greater changes in } \\
\text { waist circumference } \\
\text { and waist ratio hip } \\
(p<0.0001) \text {. An } \\
\text { intervention using a } \\
\text { low-fat, vegan diet } \\
\text { effectively reduced } \\
\text { body weight and } \\
\text { waist circumference. }\end{array}$ \\
\hline
\end{tabular}


Table 1. Cont.

\begin{tabular}{|c|c|c|c|c|c|c|}
\hline Author, Year & Population Studied & Pathology & Country & $\begin{array}{l}\text { Intervention } \\
\text { Period }\end{array}$ & $\begin{array}{c}\text { Type of } \\
\text { Intervention }\end{array}$ & Observed Outcome \\
\hline $\begin{array}{l}\text { Maruyama et al., } \\
\text { 2010 [49] }\end{array}$ & $\begin{array}{l}\mathrm{N}=99 \text { office workers } \\
\text { of the Nichirei Group } \\
\text { Corporation } \\
\text { IG: } n=52 \\
\text { M/F: } 52 / 0 \\
\text { Age Mean } \pm \mathrm{SD}= \\
43.1 \pm 7.7 \\
\text { BMI } \pm \mathrm{SD}=25.7 \pm 3.7 \\
\text { CG: } n=47 \\
\text { M/F: } 47 / 0 \\
\text { Age Mean } \pm \mathrm{SD}= \\
35.5 \pm 8.1 \\
\text { BMI } \pm \mathrm{SD}=25.8 \pm 3.3\end{array}$ & $\begin{array}{l}\text { Metabolic } \\
\text { diseases }\end{array}$ & Japan & 4 months & $\begin{array}{l}\text { IG: Individualized } \\
\text { assessment and } \\
\text { collaborative } \\
\text { goal-setting sessions } \\
\text { based on food group } \\
\text { intake and physical } \\
\text { activity, followed by } \\
\text { two individual } \\
\text { counseling sessions } \\
\text { with a registered } \\
\text { dietitian and } \\
\text { physical trainer, and } \\
\text { received monthly } \\
\text { website advice. } \\
\text { CG: No } \\
\text { intervention. }\end{array}$ & $\begin{array}{l}\text { Mean inter-group } \\
\text { differences in } \\
\text { changes were } \\
\text { significant at level } \\
p \leq 0.01 \text { for body } \\
\text { weight, BMI and } \\
\text { homeostasis model } \\
\text { assessment of } \\
\text { insulin resistance. } \\
\text { And at level } \\
p \leq 0.05 \text { for fasting } \\
\text { plasma glucose and } \\
\text { hemoglobin A1c. } \\
\text { The LiSM10! } \\
\text { program improved } \\
\text { insulin } \\
\text { resistance-related } \\
\text { metabolic } \\
\text { parameters. }\end{array}$ \\
\hline $\begin{array}{l}\text { Siegel et al., } \\
2010 \text { [50] }\end{array}$ & $\begin{array}{l}\mathrm{N}=413 \text { elementary } \\
\text { school personnel } \\
\text { IG: } n=211 \\
\text { M/F: } 35 / 176 \\
\text { Age Mean } \pm \mathrm{SE}= \\
40.0 \pm 0.73 \\
\text { BMI } \pm \mathrm{SE}=28.4 \pm 0.45 \\
\mathrm{CG}: n=202 \\
\mathrm{M} / \mathrm{F}: 53 / 149 \\
\text { Age Mean } \pm \mathrm{SE}= \\
39.5 \pm 0.84 \\
\mathrm{BMI} \pm \mathrm{SE}=27.9 \pm 0.51\end{array}$ & $\begin{array}{l}\text { Overweight } \\
\text { and obesity }\end{array}$ & USA & 3 years & $\begin{array}{l}\text { IG: Develop and } \\
\text { implement health } \\
\text { promotion activities } \\
\text { (improving diet, } \\
\text { increasing physical } \\
\text { activity, stress } \\
\text { management, etc.) } \\
\text { for employees. Each } \\
\text { intervention school } \\
\text { was given a stipend } \\
\text { of } \$ 3500 \text { per year } \\
\text { (for } 3 \text { years) to } \\
\text { subsidize its } \\
\text { wellness activities. } \\
\text { CG: Was given an } \\
\text { unrestricted stipend } \\
\text { of } \$ 1000 \text { at baseline } \\
\text { and follow-up. }\end{array}$ & $\begin{array}{l}\text { Intervention schools } \\
\text { presented a } \\
\text { significant change in } \\
\text { BMI ( } p<0.05 \text { ) but } \\
\text { not on waist-hip } \\
\text { ratio, physical } \\
\text { activity, or fruit and } \\
\text { vegetable } \\
\text { consumption. The } \\
\text { participatory } \\
\text { process appeared to } \\
\text { be an effective } \\
\text { means for } \\
\text { stimulating change. } \\
\text { The intervention } \\
\text { may have slowed } \\
\text { and perhaps } \\
\text { reversed the } \\
\text { tendency of adults } \\
\text { to gain weight } \\
\text { progressively with } \\
\text { age. }\end{array}$ \\
\hline $\begin{array}{l}\text { Van Wier et al., } \\
2009 \text { [51] }\end{array}$ & $\begin{array}{l}\mathrm{N}=1386 \text { workers from } \\
\text { two IT-companies, two } \\
\text { hospitals, an insurance } \\
\text { company, a bank and a } \\
\text { police force } \\
\text { IG phone: } n=462 \\
\mathrm{M} / \mathrm{F}=321 / 141 \\
\text { Age Mean } \pm \mathrm{SD}= \\
43 \pm 8.8 \\
\mathrm{BMI} \pm \mathrm{SD}=29.5 \pm 3.5\end{array}$ & Overweight & Netherlands & 6 months & $\begin{array}{l}\text { All groups received } \\
\text { self-help materials } \\
\text { (dealt with } \\
\text { overweight, healthy } \\
\text { diet and physical } \\
\text { activity plus } \\
\text { pedometer). } \\
\text { Additionally, the IG } \\
\text { received a lifestyle } \\
\text { intervention } \\
\text { program (10 } \\
\text { modules about } \\
\text { nutrition, physical } \\
\text { activity ... . . }\end{array}$ & $\begin{array}{l}\text { Both groups had a } \\
\text { significant decrease } \\
\text { in weight } \\
\text { loss }(p<0.001) \text { and } \\
\text { WC }(p<0.05 \\
\text { internet, } \\
p<0.001 \text { phone }) \text { in } \\
\text { comparison with the } \\
\text { CG. The difference } \\
\text { between the } \\
\text { intervention groups } \\
\text { was not statistically } \\
\text { significant. }\end{array}$ \\
\hline
\end{tabular}


Table 1. Cont.

\begin{tabular}{|c|c|c|c|c|c|c|}
\hline \multirow[t]{2}{*}{ Author, Year } & Population Studied & Pathology & Country & $\begin{array}{l}\text { Intervention } \\
\text { Period }\end{array}$ & $\begin{array}{c}\text { Type of } \\
\text { Intervention }\end{array}$ & Observed Outcome \\
\hline & $\begin{array}{l}\text { IG internet: } n=464 \\
\mathrm{M} / \mathrm{F}=302 / 162 \\
\text { Age Mean } \pm \mathrm{SD}= \\
43 \pm 8.4 \\
\mathrm{BMI} \pm \mathrm{SD}=29.6 \pm 3.4 \\
\mathrm{CG}: n=460 \\
\mathrm{M} / \mathrm{F}=306 / 154 \\
\text { Age Mean } \pm \mathrm{SD}= \\
43 \pm 8.7 \\
\mathrm{BMI} \pm \mathrm{SD}=29.6 \pm 3.7\end{array}$ & & & & $\begin{array}{l}\text { IG phone: Received } \\
\text { the program in a } \\
\text { binder. Counseling } \\
\text { by phone every two } \\
\text { weeks. } \\
\text { IG internet: Had } \\
\text { access to an } \\
\text { interactive website. } \\
\text { Counseling by email } \\
\text { when the employee } \\
\text { finished a module. } \\
\text { CG: Received only } \\
\text { the self-help } \\
\text { materials and no } \\
\text { counseling. }\end{array}$ & $\begin{array}{l}\text { Weight loss } \\
\text { intervention plus } \\
\text { lifestyle counseling } \\
\text { by phone and e-mail } \\
\text { is effective for } \\
\text { reducing body } \\
\text { weight and WC. } \\
\text { Furthermore, } \\
\text { counseling by phone } \\
\text { is effective for } \\
\text { reducing fat intake } \\
\text { and increasing } \\
\text { physical activity. }\end{array}$ \\
\hline $\begin{array}{l}\text { Leslie et al., } \\
2002 \text { [52] }\end{array}$ & $\begin{array}{l}\mathrm{N}=122 \text { petrochemical } \\
\text { work-site (staff) } \\
\text { IG1 Energy deficit diet } \\
(\mathrm{ED}): \\
n=61 \\
\mathrm{M} / \mathrm{F}: 61 / 0 \\
\text { Age Mean } \pm \mathrm{SD}= \\
41.3 \pm 8.1 \\
\mathrm{BMI} \pm \mathrm{SD}=31.5 \pm 3.7 \\
\mathrm{IG} 2 \text { Generalized low } \\
\text { calorie diet }(\mathrm{GLC}): n= \\
61 \\
\mathrm{M} / \mathrm{F}: 61 / 0 \\
\text { Age Mean } \pm \mathrm{SD}= \\
42.1 \pm 7.8 \\
\mathrm{BMI} \pm \mathrm{SD}=30.4 \pm 3.7\end{array}$ & $\begin{array}{l}\text { Overweight } \\
\text { and obesity }\end{array}$ & UK & $\begin{array}{c}12 \text { weeks } \\
\text { weight } \\
\text { loss plus } \\
12 \text { weeks } \\
\text { weight } \\
\text { mainte- } \\
\text { nance }\end{array}$ & $\begin{array}{l}\text { IG1 (ED): } \\
\text { Individualized } \\
\text { energy prescriptions } \\
\text { ( } 600 \text { kcal subtracted } \\
\text { from estimated daily } \\
\text { energy } \\
\text { requirements). Diet } \\
\text { with and without } \\
\text { meat. } \\
\text { IG2 (GLC): They } \\
\text { were given a } 1500 \\
\text { kcal eating plan. } \\
\text { Diet with and } \\
\text { without meat. } \\
\text { CG: Volunteers were } \\
\text { randomized to } \\
\text { different } \\
\text { combinations (ED } \\
\text { meat, ED no meat, } \\
\text { GLC meat, GLC no } \\
\text { meat). One-third of } \\
\text { subjects were } \\
\text { randomized to an } \\
\text { initial control period } \\
\text { prior to receiving } \\
\text { dietary advice. }\end{array}$ & $\begin{array}{l}\text { Both the ED and } \\
\text { GLC groups had a } \\
\text { significant mean } \\
\text { weight loss at week } \\
12 \text { ( } p<0.0001) \text { in } \\
\text { contrast with CG. } \\
\text { But no difference } \\
\text { was evident } \\
\text { between diet groups } \\
\text { in mean weight loss } \\
\text { at } 12 \text { weeks } \\
\text { ( } p=0.34) \text {. The } \\
\text { inclusion of lean red } \\
\text { meat in the diet did } \\
\text { not impair weight } \\
\text { loss. The weight } \\
\text { maintenance } \\
\text { intervention was not } \\
\text { effective, with a } \\
\text { significant mean } \\
\text { weight gain in all } \\
\text { groups ( } p \leq 0.003 \text { ). }\end{array}$ \\
\hline $\begin{array}{l}\text { Pritchard et al., } \\
1997 \text { [53] }\end{array}$ & $\begin{array}{l}\mathrm{N}=58 \text { business } \\
\text { corporation employees } \\
\text { IG (weight } \\
\text { loss-diet-low fat): } \\
n=18 \\
\text { Age Mean } \pm \mathrm{SD}=43.6 \\
\pm 6.0 \\
\mathrm{M} / \mathrm{F}: 18 / 0 \\
\mathrm{BMI} \pm \mathrm{SD}=29.0 \pm 2.8 \\
\mathrm{IG}(\text { weight } \\
\text { loss-exercise): } \\
n=21 \\
\text { Age Mean } \pm \mathrm{SD}= \\
44.9 \pm 6.5\end{array}$ & Overweight & Australia & 12 months & $\begin{array}{l}\text { IG (diet): Low fat } \\
\text { intake ( } 22 \% \text { to } 25 \% \\
\text { of energy) diet plus } \\
\text { personalized dietary } \\
\text { plan } \\
\text { (on the basis of } \\
\text { usual dietary } \\
\text { pattern). } \\
\text { IG (exercise): } \\
\text { Subjects selected } \\
\text { their own aerobic } \\
\text { exercise regimen } \\
\text { (realized in leisure } \\
\text { time); }\end{array}$ & $\begin{array}{l}\text { At } 12 \text { months the } \\
\text { diet group was } \\
\text { significantly } \\
\text { different from } \\
\text { baseline }(p<0.001) \\
\text { and from the CG in } \\
\text { weight lost, BMI, } \\
\text { total energy and } \\
\text { total fat mass } \\
(p<0.05) .\end{array}$ \\
\hline
\end{tabular}


Table 1. Cont.

\begin{tabular}{|c|c|c|c|c|c|c|}
\hline \multirow[t]{2}{*}{ Author, Year } & Population Studied & Pathology & Country & $\begin{array}{l}\text { Intervention } \\
\text { Period }\end{array}$ & $\begin{array}{c}\text { Type of } \\
\text { Intervention }\end{array}$ & Observed Outcome \\
\hline & $\begin{array}{l}\mathrm{M} / \mathrm{F}: 21 / 0 \\
\mathrm{BMI} \pm \mathrm{SD}=29.2 \pm 2.8 \\
\mathrm{CG}(\text { weight } \\
\text { maintenance): } \\
n=19 \\
\text { Age Mean } \pm \mathrm{SD}= \\
42.3 \pm 4.5 \\
\mathrm{M} / \mathrm{F}: 19 / 0 \\
\mathrm{BMI} \pm \mathrm{SD}=28.6 \pm 2.8\end{array}$ & & & & $\begin{array}{l}\text { minimum } \\
\text { participation (three } \\
\text { sessions of } 30 \mathrm{~min} \\
\text { per week). } \\
\text { CG: Monthly } \\
\text { weight-monitoring } \\
\text { sessions plus } \\
\text { measurement } \\
\text { protocol similar to } \\
\text { those of the } \\
\text { intervention groups } \\
\text { and followed their } \\
\text { usual pattern of } \\
\text { activity and diet. }\end{array}$ & $\begin{array}{l}\text { The dieters had } \\
\text { greater weight loss } \\
\text { than the exercise } \\
\text { group } \\
\text { (unsupervised } \\
\text { aerobic exercise) } \\
(p<0.05) \text {, as well as } \\
\text { a lower BMI index. }\end{array}$ \\
\hline $\begin{array}{c}\text { Baer } \\
1993 \text { [54] }\end{array}$ & $\begin{array}{l}\mathrm{N}=70 \\
\text { management-level } \\
\text { male employees } \\
\mathrm{IG}: n=33 \\
\mathrm{M} / \mathrm{F}=33 / 0 \\
\text { Age Mean } \pm \mathrm{SE}= \\
44 \pm 4.0 \\
\mathrm{BMI} \pm \mathrm{SD}=\mathrm{Not} \\
\text { provided } \\
\mathrm{CG}: n=37 \\
\mathrm{M} / \mathrm{F}=37 / 0 \\
\text { Age Mean } \pm \mathrm{SE}= \\
35 \pm 3.0 \\
\mathrm{BMI} \pm \mathrm{SD}=\mathrm{Not} \\
\text { provided }\end{array}$ & $\begin{array}{c}\text { Coronary } \\
\text { heart } \\
\text { disease }\end{array}$ & USA & 1 year & $\begin{array}{l}\text { All subjects met } \\
\text { with a registered } \\
\text { dietitian who } \\
\text { explained the results } \\
\text { of the lipid analysis } \\
\text { and discussed risk } \\
\text { factors for coronary } \\
\text { heart disease with } \\
\text { an emphasis on diet. } \\
\text { IG: Nutrition } \\
\text { intervention: } \\
\text { individualized } \\
\text { instruction about the } \\
\text { step } 1 \text { diet; group } \\
\text { sessions (1 h every } \\
3 \text { months) on eating } \\
\text { out, dietary fiber, } \\
\text { and maintaining } \\
\text { heart-healthy } \\
\text { behaviors, and } \\
\text { individualized } \\
\text { follow-up by } \\
\text { telephone (one call } \\
\text { per month). }\end{array}$ & $\begin{array}{l}\text { Significant decreases } \\
(p<0.05) \text { in total } \\
\text { cholesterol, } \\
\text { triglycerides, body } \\
\text { weight and body fat } \\
\text { were observed in } \\
\text { intervention subjects } \\
\text { at the 1-year } \\
\text { follow-up. } \\
\text { Although weight } \\
\text { reduction was not a } \\
\text { goal of the program, } \\
\text { by decreasing } \\
\text { energy intake and } \\
\text { increasing energy } \\
\text { expenditure, } \\
\text { subjects lost weight } \\
\text { and decreased body } \\
\text { fat. } \\
\text { The worksite } \\
\text { provides many } \\
\text { opportunities for } \\
\text { dietetics } \\
\text { professionals to } \\
\text { conduct nutrition } \\
\text { education programs } \\
\text { to decrease risk } \\
\text { factors associated } \\
\text { with coronary heart } \\
\text { disease. }\end{array}$ \\
\hline $\begin{array}{l}\text { Follick et al., } \\
1984 \text { [55] }\end{array}$ & $\begin{array}{l}\mathrm{N}=48 \text { employees of a } \\
\text { general hospital } \\
\text { M/F: } 41 / 7 \\
\text { Age range: } 20-69 \\
\text { IG: } n=24\end{array}$ & Overweight & USA & 18 weeks & $\begin{array}{l}\text { IG: Weight loss } \\
\text { program }(14 \text { session } \\
\text { behavior } \\
\text { modification } \\
\text { program) plus } \\
\text { incentive procedure. } \\
(5 \$(\times 14) \text { deposit } \\
\text { was returned (one } \\
\text { for each treatment } \\
\text { session). }\end{array}$ & $\begin{array}{l}\text { Both groups lost } \\
\text { weight over the } \\
\text { course of the } \\
\text { intervention } \\
(p<0.001) \text { and there } \\
\text { were no significant } \\
\text { differences in } \\
\text { weight loss between } \\
\text { groups. }\end{array}$ \\
\hline
\end{tabular}


Table 1. Cont.

\begin{tabular}{|c|c|c|c|c|c|c|}
\hline Author, Year & Population Studied & Pathology & Country & $\begin{array}{l}\text { Intervention } \\
\text { Period }\end{array}$ & $\begin{array}{c}\text { Type of } \\
\text { Intervention }\end{array}$ & Observed Outcome \\
\hline & $\begin{array}{l}\mathrm{BMI} \pm \mathrm{SD}=\text { Not } \\
\text { provided } \\
\mathrm{CG}: n=24 \\
\mathrm{BMI} \pm \mathrm{SD}=\text { Not } \\
\text { provided }\end{array}$ & & & & $\begin{array}{l}\text { CG: Weight loss } \\
\text { program alone. }\end{array}$ & $\begin{array}{l}\text { The inclusion of an } \\
\text { incentive procedure } \\
\text { may improve the } \\
\text { effectiveness of a } \\
\text { behavioral weight } \\
\text { loss intervention by } \\
\text { decreasing attrition } \\
(p<0.01) \text {. }\end{array}$ \\
\hline
\end{tabular}

BMI: Body mass index; CG: Control group; DI: Dietary intervention; ED: Energy deficit diet; EDM: Environmental dietary modification; GLC: Generalized low-calorie diet; IG: Intervention group; IP: Incentivized participants; lbs: pound-weight; LMW: Livin' my weigh; M/F = Male/Female; MS: Metabolic syndrome; NE: Nutrition education; NIP: Non incentivized participants; NP: Not provided; PA: Physical activity; SD: Standard deviation; SE: Standard error; TMC: Telemedicine coaching; WC: Waist circumference; WM; Weight management.

The agreement among the evaluators regarding the relevance of selected studies, calculated using the KI, was $74.88 \%(p=0.01)$.

The selected articles had an actuality, as determined by the BK, equal to 7.50 years, with a PI of $29.41 \%$. The years with the highest number of published works were 2012, 2015 and 2017, with four trials published each of those years; see Table 1.

When evaluating the transparency of reporting of the trials selected for the review, the CONSORT checklist scores ranged from a minimum of 3.50 ( $14 \%$ compliance) to a maximum of 20.50 ( $82.50 \%$ compliance) with a median of 12.75 (Table 2), observing, across time, a good increasing exponential trend $\left(R^{2}=0.62 ; p<0.001\right)$.

Table 2. Assessment of study quality according to the 25-item CONSORT guidelines.

\begin{tabular}{|c|c|c|c|c|c|c|c|c|c|c|c|c|c|c|c|c|c|c|c|c|c|c|c|c|c|c|}
\hline A & 1 & & 3 & 4 & & 6 & 7 & 8 & 9 & 10 & 11 & 12 & 13 & 14 & 15 & 16 & 17 & 18 & 19 & 20 & 21 & 22 & 23 & 24 & 25 & Total\% \\
\hline Thornd & 1 & & 05 & 1 & & 05 & 0.5 & 1 & 0 & 0 & 0.5 & 1 & 0.5 & 0.5 & 1 & 1 & 0.5 & 1 & 0 & 1 & 0 & 1 & 1 & 0 & 0 & 5.562 \\
\hline Röhl & 1 & 1 & 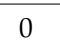 & 1 & & & 0.5 & 1 & & & 0.5 & 1 & 1 & 0.5 & 1 & 1 & 0.5 & 1 & 1 & 1 & 1 & & 1 & 1 & 1 & 582 \\
\hline Itur & 1 & 1 & 0 & 0.5 & 0 & 0. & 0 & 0.5 & 0 & 0 & 0.5 & 0.5 & 1 & 0.5 & 0 & 1 & 0.5 & 0 & 0 & 0 & 0 & 1 & 1 & 1 & 1 & \\
\hline Day & 1 & 1 & 0 & 0.5 & 0 & 0.5 & 0. & 0 & 0 & 0 & 0 & 0 & 1 & 0.5 & 1 & 1 & 0.5 & 0 & 0 & 1 & 0 & 1 & 1 & 0 & 0 & 1.546 \\
\hline $\mathrm{Ke}$ & 1 & 1 & 0 & 0.5 & & 05 & 0. & 1 & 1 & 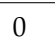 & 0.5 & 0.5 & 1 & 0.5 & 1 & 1 & 0.5 & 0 & 1 & 1 & 1 & 1 & 1 & o & 1 & .566 \\
\hline Tene & 1 & 1 & 0.3 & 0.6 & & דיט. & 0 & 1 & . & 0 & 0.5 & 1 & 0 & 0.5 & 1 & 0 & 0.5 & 0 & 0 & 1 & 0 & 1 & 1 & 0 & 1 & 13 \\
\hline Vieste & 1 & 1 & 0.5 & 0.5 & 1 & 0.5 & 0.5 & 0.5 & 0 & 1 & 0.5 & 0.5 & 1 & 0 & 1 & 0 & 0.5 & 0 & 0 & 1 & 0 & 1 & 1 & 0 & 0 & 13 \\
\hline Shri & 1 & 1 & 0 & 0 & 1 & 0 & 0 & 0.5 & 0 & 0 & 0 & 0.5 & 1 & 0.5 & 1 & 1 & 0.5 & 0 & 0 & 1 & 0 & 1 & 1 & 0 & 1 & 56 \\
\hline $\mathrm{Ge}$ & 1 & 1 & 0.5 & 0.5 & 1 & 05 & 0. & 1 & 0 & 0 & 0.5 & 0.5 & 1 & 0.5 & 1 & 1 & 0.5 & 0 & 0 & 1 & 0 & 1 & 1 & o & 1 & 0 \\
\hline $\mathrm{F}$ & ? & 1 & 0 & 0.5 & 1 & 0.3 & 0 & 0 & c & 0 & 0 & 0.5 & 0 & 0 & 1 & 0 & 0.5 & 0 & 0 & 1 & 0 & 1 & 0 & 0 & 1 & 6 \\
\hline Gean & 1 & 1 & 0.5 & 0.5 & 0 & 0.5 & 0.5 & 0.5 & 0 & 1 & 0 & 0.5 & 1 & 0 & 1 & 1 & 0.5 & 0 & 0 & 1 & 1 & 1 & 1 & 1 & 1 & 15.562 \\
\hline Sole & 1 & 1 & 0 & 0 & 1 & 0.5 & 0 & 0 & 0 & 0 & 0 & 0 & 1 & 0 & 1 & 1 & 0.5 & 0 & 0 & 1 & 0 & 1 & 0 & 0 & 1 & 10 \\
\hline Mit & 1 & 1 & 0.5 & 0.5 & 1 & 0.5 & 0 & 0.5 & 0 & 0 & 0.5 & 0.5 & 1 & 0 & 1 & 1 & 0.5 & 0 & 0 & 1 & 1 & 1 & 1 & 0 & 1 & 62 \\
\hline Ferna & 1 & 1 & 0.5 & 0.5 & 1 & 0.5 & 0 & 0 & 0 & 0 & 0.5 & 0.5 & 1 & 0.5 & 1 & 1 & 0.5 & 0 & 0 & 1 & 1 & 1 & 0 & 0 & 1 & $13.5 \quad 64$ \\
\hline Alme & 1 & 1 & 1 & 1 & 0 & 0.5 & 0 & 1 & 0 & 0 & 0 & 0.5 & 1 & 0.5 & 1 & 1 & 0.5 & 0 & 0 & 1 & 1 & 1 & 1 & 1 & 1 & 16 \\
\hline$\varnothing$ & 05 & 1 & 1 & 1 & 1 & 05 & 0 & 0.5 & 0 & 0 & 0 & 0.5 & 0.5 & 0.5 & 1 & 1 & 0.5 & 0 & 0 & 1 & 0 & 1 & 1 & 0 & 1 & 14 \\
\hline $\mathrm{V}$ & 0.5 & 1 & 0 & 0.5 & 1 & 0.5 & 0 & 0 & 0 & 0 & 0 & 0.5 & 1 & 0.5 & 1 & 1 & 0.5 & 0 & 0 & 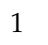 & 0 & 1 & 1 & 0 & 1 & 13 \\
\hline Misl & 1 & 1 & 0 . & 1 & 1 & 0.5 & 0.5 & 0.5 & 0 & 0 & 0 & 0.5 & 1 & 0 & 1 & 1 & 0.5 & 0 & 0 & 1 & 0 & 1 & 0 & 0 & 0 & 12 \\
\hline Salin & 0.5 & 1 & . & 0 & 0 & 0.5 & 0 & 0.5 & 0 & 0 & 0 & 1 & 1 & 0.5 & 1 & 1 & 0.5 & 1 & 0 & 0 & 0 & 1 & 0 & 0 & 0 & 9.5 \\
\hline Christensen $\mathrm{e}$ & 1 & 1 & 0.5 & 0.5 & 1 & 0.0 & . & 0.5 & 1 & 1 & 0.5 & 0.5 & 1 & 0.5 & 0 & 1 & 0.5 & 0 & 0 & 1 & 0 & 1 & 1 & 0 & 1 & 15 \\
\hline Thorr & 1 & 1 & 0 & 1 & 0 & 0.0 & 0.5 & 0.5 & 0 & U & 0 & 1 & 1 & 0 & 1 & 1 & 0.5 & 0 & 0 & 1 & 0 & 1 & 1 & 0 & 1 & 10 \\
\hline Linde & 1 & 1 & 0.5 & 0.5 & 1 & 0. & 0 & 1 & 0 & 1 & 0 & 0.5 & 1 & 0 & 1 & 1 & 0.5 & 0 & 0 & 1 & 0 & 1 & 1 & 0 & 1 & 14 \\
\hline Nanr & 1 & 1 & 0.5 & 0.5 & 0 & 0.5 & 0 & 0 & 0 & 0 & 0 & 0.5 & 1 & 0 & 1 & 1 & 0.5 & 0 & 0 & 1 & 1 & 1 & 1 & 0 & 1 & 12.550 \\
\hline
\end{tabular}


Table 2. Cont.

\begin{tabular}{lllllllllllllllllllllllllllll}
\hline \multicolumn{1}{c}{ Author } & $\mathbf{1}$ & $\mathbf{2}$ & $\mathbf{3}$ & $\mathbf{4}$ & $\mathbf{5}$ & $\mathbf{6}$ & $\mathbf{7}$ & $\mathbf{8}$ & $\mathbf{9}$ & $\mathbf{1 0}$ & $\mathbf{1 1}$ & $\mathbf{1 2}$ & $\mathbf{1 3}$ & $\mathbf{1 4}$ & $\mathbf{1 5}$ & $\mathbf{1 6}$ & $\mathbf{1 7}$ & $\mathbf{1 8}$ & $\mathbf{1 9}$ & $\mathbf{2 0}$ & $\mathbf{2 1}$ & $\mathbf{2 2}$ & $\mathbf{2 3}$ & $\mathbf{2 4}$ & $\mathbf{2 5}$ & Total\% \\
\hline Brehm et al. [45] & 1 & 1 & 0.5 & 0.5 & 1 & 0.5 & 0 & 0.5 & 0 & 0 & 0 & 0.5 & 1 & 0 & 0 & 1 & 0.5 & 0 & 0 & 0 & 0 & 1 & 0 & 0 & 0 & 9 & 36 \\
\hline Christensen et al. [46] & 1 & 1 & 0.5 & 0.5 & 1 & 0.5 & 0.5 & 0.5 & 1 & 1 & 0.5 & 0.5 & 1 & 0 & 1 & 1 & 0.5 & 0 & 0 & 0 & 1 & 1 & 1 & 0 & 0 & 12 & 48 \\
\hline Barham et al. [47] & 0.5 & 1 & 0 & 0 & 1 & 0.5 & 0 & 0 & 0 & 0 & 0 & 0.5 & 0.5 & 0 & 1 & 0 & 0.5 & 0 & 0 & 0 & 0 & 1 & 0 & 0 & 0 & 6.5 & 26 \\
\hline Ferdowsian et al. [48] & 0.5 & 0.5 & 0 & 0.5 & 0 & 0 & 0 & 0 & 0 & 0 & 0 & 0.5 & 1 & 0 & 0 & 1 & 0.5 & 0 & 1 & 1 & 0 & 0 & 1 & 0 & 1 & 8.5 & 34 \\
\hline Maruyama et al. [49] & 1 & 1 & 0.5 & 0 & 0 & 0.5 & 0 & 0.5 & 0 & 1 & 0 & 0.5 & 1 & 0.5 & 1 & 1 & 0.5 & 0 & 0 & 1 & 0 & 1 & 0 & 0 & 1 & 13 & 52 \\
\hline Siegel et al. [50] & 1 & 1 & 0 & 0 & 0 & 0.5 & 0 & 0 & 0 & 0 & 0 & 0 & 0.5 & 0 & 1 & 1 & 0.5 & 0 & 0 & 0 & 1 & 1 & 0 & 0 & 1 & 9.5 & 38 \\
\hline Van Wier et al. [51] & 1 & 1 & 1 & 0.5 & 0.5 & 0.5 & 0.5 & 0.5 & 0 & 0 & 0 & 0.5 & 1 & 0 & 1 & 1 & 0.5 & 0 & 0 & 1 & 0 & 1 & 1 & 0 & 1 & 15 & 56 \\
\hline Leslie et al. [52] & 0.5 & 1 & 0.5 & 0.5 & 0.5 & 0.5 & 0.5 & 0 & 1 & 0 & 0 & 0.5 & 0.5 & 0 & 1 & 0 & 0.5 & 0 & 0 & 0 & 0 & 1 & 0 & 0 & 1 & 9 & 36 \\
\hline Pritchard et al. [53] & 0.5 & 1 & 0 & 0 & 0 & 0.5 & 0 & 0 & 0 & 0 & 0 & 1 & 1 & 0 & 1 & 1 & 0.5 & 0 & 0 & 0 & 0 & 1 & 0 & 0 & 1 & 8.5 & 34 \\
\hline Baer [54] & 1 & 0.5 & 0 & 0 & 0 & 0.5 & 0 & 0 & 0 & 0 & 0 & 0.5 & 0 & 0 & 1 & 0 & 0.5 & 0 & 0 & 0 & 0 & 1 & 0 & 0 & 0 & 6 & 24 \\
\hline Follick et al. [55] & 0.5 & 1 & 0 & 0 & 0 & 0.5 & 0 & 0 & 0 & 0 & 0 & 0 & 0 & 0 & 0 & 0 & 0.5 & 0 & 0 & 0 & 0 & 1 & 0 & 0 & 0 & 3.5 & 14 \\
\hline
\end{tabular}

Based on the SIGN criteria, this review presented evidence with a grade of 1- (systematic review of randomized clinical trials or randomized clinical trials with a high risk of bias) with a recommendation grade of B (a body of evidence that includes studies directly applicable to the target population and that demonstrates global consistency of the results or the extrapolation of studies rated as 1).

The majority of the studies included in the review were from the USA, with 17 trials $[22,25,31,34-37,39,40,42,43,45,47,48,50,54,55]$ and the Netherlands, with three trials $[28,38,51]$.

The study with the largest population was that by Fernández et al. [35], with $n=2614$ workers, and the study with the smallest population was that by Almeida et al. [36], with 28 workers. All participants were of working age (between 18 and 65 years).

The mean body mass index (BMI) in the intervention group fluctuated between a minimum of $23.8 \pm 3.5$ in the study by Iturriaga et al. [24] and a maximum of $39.4 \pm 6.9$ in the study by Barham et al. [47]. There were four clinical trials that did not report BMI: Shrivastava et al. [29] only indicated the percent of obese individuals; Follick et al. [55] only included the percent of overweight individuals; Baer [54] only reported weight in kilograms and Ferdowsian et al. [48] did not report any measure related to BMI.

The main pathologies observed in the study population were overweight and obesity [22-25,35,36,38,40-43,46,50,52]; overweight [26,29,53,55]; obesity [37,45,51]; overweight, obesity and diabetes [31,32,34,39,47,48]; abdominal obesity and dyslipidemia [27,30]; overweight and musculoskeletal disorders [28]; obesity, diabetes and cardiovascular disease [33]; metabolic syndrome [44]; metabolic disease [49], and metabolic disease [54].

The intervention period ranged from a minimum of 10 weeks [34,42] to a maximum of 3 years [50], with 12 months being the most frequent intervention period $[22,26,36,37,40,41,45,46,53,54]$

\subsection{Types of Interventions Performed}

Due to the heterogeneity of the actions carried out, in the clinical trials analyzed, the different interventions carried out were grouped into the following seven categories:

1. Dietary interventions associated with other actions (exercise or educational program): seven studies [23,27,30,46,48,52,53].

2. Interventions related to the workplace environment, including educational actions, financial incentives, availability and price of food and portion control: five studies $[22,32,35,43,45]$.

3. Exclusive educational interventions aimed toward lifestyle, dietetics, physical activity, and stress management, including televigilance devices and counseling: 16 studies $[25,26,28,29,33,34,36,37,40-42,44,47,49,51,54]$. 
4. Economic incentives added to training actions aimed at weight loss, physical activity and dieting: three studies [31,50,55].

Multicomponent intervention, through the combination of mindfulness, e-coaching and the addition of fruits and vegetables: one study [38]; or dietary intervention (facilitating a greater supply of food in cafeterias): one study [39]; or intervention focused on physical exercise: one study [24].

\subsection{Main Results Derived from the Interventions}

From the interventions developed, the following results could be verified:

Dietary interventions associated with other actions (mainly physical exercise) decreased body weight in the intervention group [27,46,53]. Gepner et al. [30] also observed improvements in cardiometabolic markers, and Ferdowsian et al. [48], along with weight loss, reported a decrease in waist circumference. The intervention program implemented by Röhling et al. [23] (the SAMMAS intervention) achieved long-term weight loss maintenance. In contrast, in a previous trial, Leslie et al. [52] concluded that the body weight maintenance intervention was not effective.

Behavioral environmental strategies improved food selection, which, according to Thorndike et al. [22], resulted in improvements in body weight. Even educational actions were effective in promoting healthy diets [32] and were postulated as promising long-term interventions (2 years) [35]. However, Linde et al. [43] and Brehm et al. [45] indicated that environmental changes in the workplace were not enough to improve the weight and health of workers.

Educational interventions showed their suitability for implementation in the workplace; such interventions resulted in a decrease in weight and BMI in the treatment group [25,29,36,41,47]. Kempf et al. [26] also observed a decrease in BMI in the intervention group, but their results were not supported by intention-to-treat analysis. Mitchell et al. [34] confirmed that weight loss was associated with greater attendance at educational intervention sessions. Likewise, educational actions improve metabolic parameters [49], cardiometabolic risk factors [40] and the prevalence of metabolic syndrome [44]. Furthermore, this type of intervention was shown to be valid in improving the risks associated with coronary disease [54].

For the follow-up of these training activities, van Wier et al. [51] demonstrated that telephone follow-up was effective. However, Solenhill et al. [33] found that telephone counseling did not have positive effects on employees, and Thorndike et al. [42] concluded that online support was not effective.

Viester et al. [28] concluded that these actions could have promising long-term effects, but differences between the intervention and control groups were not significant. The study by Østbye et al. [37] found no differences related to the implementation of an educational program.

The use of economic incentives as the main intervention influenced the weight loss of participants [31] and even decreased attrition [55]. It was also effective in stimulating change toward healthier attitudes, reducing the tendency to increase body weight [50]. However, no study showed results related to the period after the incentive ceases.

Other multicomponent actions [38] (combination of mindfulness, e-coaching and the addition of fruits and vegetables) did not show clear causal effects at 6 and 12 weeks.

Exclusive dietary action (a low-fat vegan diet) [39] produced an improvement in body weight at 18 weeks, but results for the maintenance period and long-term results were not indicated.

For an intervention focused on physical exercise [24], beneficial effects on body composition were observed in the short term (12 weeks), but postintervention results were not included.

\subsection{Main Results Derived of Meta-Analysis}

For the meta-analysis, 35 groups of 22 articles were included. 


\section{- $\quad$ Effect size}

The effect sizes calculated from the meta-analysis are shown in Figure 2 as well as tests for the presence of heterogeneity.

\section{Study}

Röhling et al.

Christensen et al.

Leslie et al.

Leslie et al.

Pritchard et al.

Pritchard et al.

Thorndike et al.

Thorndike et al.

Geaney et al.

Geaney et al.

Geaney et al.

Fernández et al.

Brehm et al.

Brehm et al.

Brehm et al.

Viester et al.

Viester et al.

Shrivastava et al.

Solenhill et al.

Solenhill et al.

Mitchell et al

Mitchell et al

Almeida et al.

Almeida et al.

$\varnothing$ stbye et al.

$\varnothing$ øtbye et al.

Salinardi et al.

Christensen et al.

Thorndike et al.

Thorndike et al.

Nanri et al.

Maruyama et al.

Siegel et al.

Mishra et al.

Iturriaga et al.

Random effects model

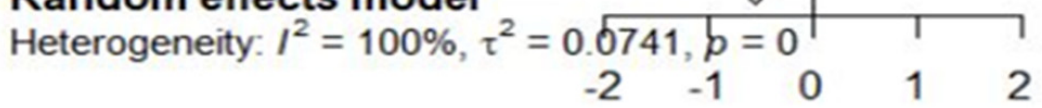

Mean MRAW

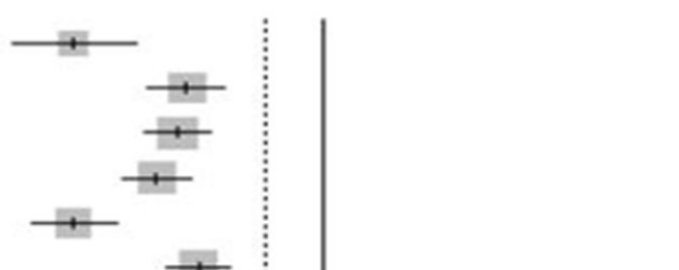

$-2.40[-3.00 ;-1.80]$

$-1.31[-1.68 ;-0.94]$

$-1.40[-1.71 ;-1.09]$

$-1.60[-1.93 ;-1.27]$

$-2.40 \quad[-2.82 ;-1.98]$

$-1.20[-1.50 ;-0.90]$

0.20 [ $0.00 ; 0.40$ ]

$0.50 \quad[0.20 ; 0.80]$

$-0.20 \quad[-0.39 ;-0.01]$

$-0.10 \quad[-0.33 ; 0.13]$

$-0.30 \quad[-0.40 ;-0.20]$

$-0.51 \quad[-0.53 ;-0.49]$

$0.50 \quad[0.45 ; 0.55]$

$0.50 \quad[0.45 ; 0.55]$

0.60 [ $0.55 ; 0.65]$

0.20 [ $0.13 ; 0.27]$

$0.20 \quad[0.13 ; 0.27]$

$-0.61[-0.76 ;-0.46]$

$-0.10[-0.14 ;-0.06]$

$0.10 \quad[0.07 ; 0.13]$

$-0.80[-1.30 ;-0.30]$

$-1.20[-1.40 ;-1.00]$

$-0.36[-0.36 ;-0.36]$

$-0.20[-0.21 ;-0.19]$

$-0.36[-0.66 ;-0.06]$

$-0.25[-0.53 ; 0.03]$

$-2.80[-2.84 ;-2.76]$

$-2.20[-2.51 ;-1.89]$

$-0.80[-0.90 ;-0.70]$

$-0.50 \quad[-0.60 ;-0.40]$

$-0.67[-0.81 ;-0.53]$

$-0.74[-1.01 ;-0.47]$

$-0.04[-0.04 ;-0.04]$

$-1.50[-1.54 ;-1.46]$

$-0.27[-0.55 ; 0.01]$
$1.5 \%$

$2.2 \%$

$2.5 \%$

$2.4 \%$

$2.1 \%$

$2.5 \%$

$2.9 \%$

$2.5 \%$

$2.9 \%$

$2.8 \%$

$3.2 \%$

$3.3 \%$

$3.3 \%$

$3.3 \%$

$3.3 \%$

$3.2 \%$

$3.2 \%$

$3.1 \%$

$3.3 \%$

$3.3 \%$

$1.8 \%$

$2.9 \%$

$3.3 \%$

$3.3 \%$

$2.5 \%$

$2.6 \%$

$3.3 \%$

$2.5 \%$

$3.2 \%$

$3.2 \%$

$3.1 \%$

$2.6 \%$

$3.3 \%$

$3.3 \%$

$2.6 \%$

\section{$-0.55[-0.65 ;-0.46] 100.0 \%$}

Figure 2. Forest plot for Body Mass Index.

- Heterogeneity of included studies

The included studies show strong heterogeneity (100\%). Table 3 shows the effect of each study on the total heterogeneity. We observe that none of them is very influential. Similarly, we can see in Figure 3 of the Baujat graph that no study is in the upper right corner. 
Table 3. Summary leave-one-out, Baseline of Body Mass Index and interventions.

\begin{tabular}{|c|c|c|c|c|c|c|c|c|c|}
\hline ID & Autor & $\begin{array}{c}\text { Change } \\
\text { Effect }\end{array}$ & $\begin{array}{c}\text { Baseline } \\
\text { BMI }\end{array}$ & Int & ID & Autor & $\begin{array}{c}\text { Change } \\
\text { Effect }\end{array}$ & $\begin{array}{c}\text { Baseline } \\
\text { BMI }\end{array}$ & Int \\
\hline 1 & Röhling et al. & 0.53 & 3 & 1 & 19 & Solenhill et al. & 0.57 & 2 & 3 \\
\hline 2 & Christensen et al. & 0.54 & 3 & 1 & 20 & Solenhill et al. & 0.58 & 2 & 3 \\
\hline 3 & Leslie et al. & 0.53 & 3 & 1 & 21 & Mitchell et al. & 0.55 & 2 & 3 \\
\hline 4 & Leslie et al. & 0.53 & 3 & 1 & 22 & Mitchell et al. & 0.53 & 2 & 3 \\
\hline 5 & Pritchard et al. & 0.52 & 2 & 1 & 23 & Almeida et al. & 0.58 & 3 & 3 \\
\hline 6 & Pritchard et al. & 0.54 & 2 & 1 & 24 & Almeida et al. & 0.58 & 3 & 3 \\
\hline 7 & Thorndike et al. & 0.58 & 2 & 2 & 25 & Østbye et al. & 0.56 & 3 & 3 \\
\hline 8 & Thorndike et al. & 0.58 & 2 & 2 & 26 & Østbye et al. & 0.56 & 3 & 3 \\
\hline 9 & Geaney et al. & 0.57 & 2 & 2 & 27 & Salinardi et al. & 0.46 & 3 & 3 \\
\hline 10 & Geaney et al. & 0.57 & 2 & 2 & 28 & Christensen et al. & 0.51 & 3 & 3 \\
\hline 11 & Geaney et al. & 0.56 & 2 & 2 & 29 & Thorndike et al. & 0.55 & 2 & 3 \\
\hline 12 & Fernández et al. & 0.55 & 2 & 2 & 30 & Thorndike et al. & 0.56 & 2 & 3 \\
\hline 13 & Brehm et al. & 0.59 & 2 & 2 & 31 & Nanri et al. & 0.55 & 2 & 3 \\
\hline 14 & Brehm et al. & 0.59 & 2 & 2 & 32 & Maruyama et al. & 0.55 & 2 & 3 \\
\hline 15 & Brehm et al. & 0.59 & 2 & 2 & 33 & Siegel et al. & 0.59 & 2 & 4 \\
\hline 16 & Viester et al. & 0.58 & 2 & 3 & 34 & Mishra et al. & 0.52 & 3 & 5 \\
\hline 17 & Viester et al. & 0.58 & 2 & 3 & 35 & Iturriaga et al. & 0.56 & 1 & 5 \\
\hline 18 & Shrivastava et al. & 0.55 & 2 & 1 & Pooled & & 0.55 & & \\
\hline
\end{tabular}

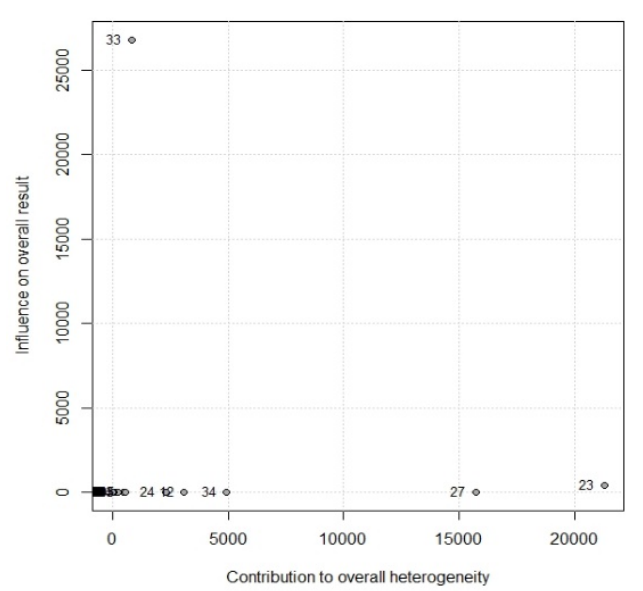

(a)

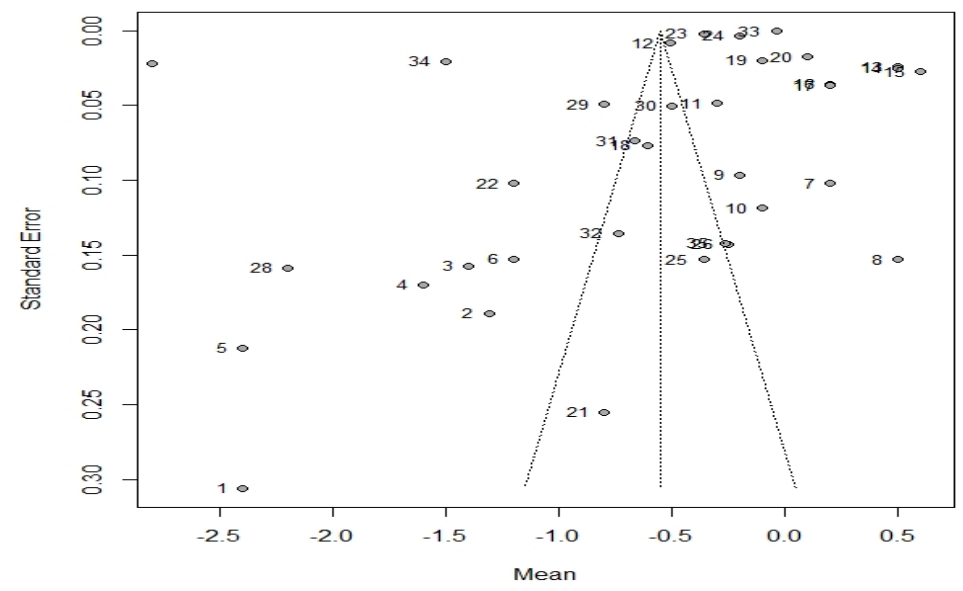

(b)

Figure 3. (a) Baujat plot for Body Mass Index (b) Funnel plot for Body Mass Index.

The Bajujat graph (Figure 3a) shows that no single group has a decisive weight on the outcome of the meta-analysis. In fact, in the leave-one-out test, no study varied heterogeneity by more than $1 \%$.

- Heterogeneity of non-included studies (publication bias)

Another source of heterogeneity could come from publication bias. For this purpose, we analyze the symmetry of the Funnel-polt (Figure 3b).

As can be seen, there is not much symmetry, so publication bias may be high. The results of the Trim-fill and Copas techniques suggest that many unpublished studies, between 12 and 48 respectively, would be needed to compensate for this lack of symmetry. The model proposed by Copas would reduce the size of the effect on BMI but still be significant, in the case of the Trim-fill adjustment this reduction would end up not being statistically significant.

- Moderator analysis (meta-regression) 
Other sources of possible heterogeneity may be the influence of covariates or moderators. Table 4 studies the effects of the five types of interventions and the baseline BMI.

Table 4. Moderator analysis, adjusted meta-regression by the baseline of Body Mass Index and interventions.

\begin{tabular}{ccccccccccccc}
\hline Variable & Baseline BMI & Sig. & Int-1 & Sig. & Int-2 & Sig. & Int-3 & Sig. & Int-4 & Sig. & Int-5 & Sig. \\
\hline INTERCEPT & 1.36 & $<0.01$ & -0.39 & $<0.01$ & -0.81 & $<0.01$ & -0.52 & $<0.01$ & -0.59 & $<0.01$ & -0.52 & $<0.01$ \\
\hline Coef & -0.85 & $<0.01$ & -1.26 & $<0.01$ & 0.94 & $<0.01$ & -0.11 & 0.37 & 0.55 & 0.12 & -0.44 & 0.03 \\
\hline Adjusted & Baseline BMI & Sig. & Int-1 & Sig. & Int-2 & Sig. & Int-3 & Sig. & Int-4 & Sig. & Int-5 & Sig. \\
\hline INTERCEPT & 0.47 & 0.16 & & & & & & & & & & \\
\hline Coef & -0.48 & $<0.01$ & -0.87 & $<0.01$ & 0.62 & $<0.01$ & 0.13 & 0.59 & 0.48 & 0.21 & -0.46 & 0.08 \\
\hline
\end{tabular}

There is variation in the effect size of BMI by baseline. Interventions give better results on obese groups than on groups with overweight or normal weight. As for the interventions, 1 and 2 were statistically significant in the multivariate model but with opposite directions. Intervention 1 decreases BMI and intervention 2 increases BMI.

\section{Discussion}

Following the recommendations regarding the objectives of a systematic review [56], the current review synthesized the relevant information related to nutrition, food and diet interventions implemented in occupational health to provide the scientific community with relevant information that can help promote new interventions for workers protection. In addition, this study is part of the strategy of the World Health Organization that emphasizes the importance of establishing primary prevention and interventions aimed at improving occupational health [57].

It could be considered that the most prevalent occupational disease (although it is not considered as such) is undoubtedly obesity (and overweight) because it affects many workers [58] and those who are overweight or obese are more likely to suffer injuries than normal-weight workers [59].

The analysis of the actuality of the reviewed studies demonstrated the full validity of the selected studies because the data obtained showed greater relevance than what was calculated for the bibliometric studies in fields related to the sciences of nutrition and occupational health [4] and more current than that found for recent systematic reviews related to occupational health $[60,61]$.

The evaluation of the reporting transparency of the studies included in the review articles, as assessed by the CONSORT criteria, was similar to that for other review articles $[62,63]$. The analysis of the progression of documentary adequacy that was observed in the most current articles was mainly due to the implementation of CONSORT criteria. In fact, the oldest works did not usually follow these quality guidelines; for example, the first documents that used the CONSORT criteria date back to 1996 [64], and their use was progressive. If clinical trials have an inadequate methodology or, especially, if the final description of the trial does not contain certain information, readers cannot adequately judge the validity of the study, and the scientific evidence related to the results is very limited [65].

The level of evidence and grade of recommendation for this study, as determined using the SIGN criteria, were consistent or even better than those observed in previous studies. Despite seeking a consistent cause-effect relationship, because intervention studies were sought, some were subject to more bias than others and therefore, more weakly support the conclusions [66]. The conclusions of many studies of occupational health and safety are still not based on the greatest possible evidence [67]. This may be due to the experimental design of primary studies, such as clinical trials, which are considered robust but may not be adequate to evaluate interventions in occupational health when presenting, generally, very long-term effects; furthermore, as in this review, nutritional interventions 
were not the most studied mediations in relation to work and were more oriented to combat certain diseases.

All the studies focused primarily on overweight and/or obese populations, except for the studies by Nanri et al. [44], which focused on a population with metabolic syndrome, that by Maruyama et al. [49], which focused on metabolic disease in general and that by Baer [54], which focused on heart disease.

BMI was provided in the vast majority of the studies, and it was considered that those that did not provide a clear measure to evaluate interventions $[29,48,54,55]$ could not have adequately reported their results. This inadequate description of clinical studies can be, in any case, a waste of time for those who seek valid information derived from clinical trials [65]. The lack of information in a publication can result in the work being excluded when carrying out a systematic review on a certain intervention. Approximately one-third of clinical trials can be excluded from systematic reviews because relevant data are lacking [68]. In this review, it was decided to retain these four clinical trials to provide as much information as possible but not dismiss the lack of relevant results.

Dietary-nutritional interventions within companies are always complex due to the idiosyncrasy of the workforce and, generally, the short period available to perform these interventions [69]. Thus, the follow-up period must be adequate to assess the results of the intervention, a requirement that all the selected trials met. A period of several weeks, even months, is considered necessary to assess the results [61,70].

In general, interventions using any mode of interaction (face-to-face, telephone, internet, etc.), directed by a trained professional, were effective in improving outcomes related to overweight and obesity.

From the interventions observed, it was possible to deduce that the actions that included several strategies achieved adequate results in the working population. This statement is consistent with the results reported by Upadhyaya et al. [71], who concluded that occupational health professionals should continue to be creative in the development of multicomponent interventions (combining behavioral/educational, environmental and organizational support).

The effectiveness of dietary interventions associated with other actions (mainly physical exercise) is a well-known topic. Their effectiveness in the management of obesity and overweight in the work environment has already been demonstrated [72,73]. However, the structures and cultures of the workplace should always be considered when planning interventions. The negotiation and flexibility of stakeholders play essential roles in overcoming resistance to change [74].

Among the combined strategies, environmental interventions have been proposed as appropriate actions for the promotion of healthy habits, although they were not considered sufficient, by themselves, to improve the weight and health of workers $[43,45]$. Thus, the review by Chu et al. [75], confirmed the consistency of the effectiveness of multicomponent environmental interventions.

The results obtained showed a causal relationship when implementing educational measures in the workplace focused on decreasing body weight, resulting in improvements in metabolic parameters [49], cardiometabolic risk factors [40] and prevalence of metabolic syndrome [44]. This type of intervention was shown to be valid in improving the risks associated with coronary disease [54]. However, the review by Wolfenden et al. [76] concluded that it was not clear whether such strategies were profitable or generated unintended adverse consequences, thus justifying more research to seek more evidence.

The strategies that included financial incentives (generally discounts for healthy items on the menu for the company cafeteria) when choosing the healthiest menu items were shown to be effective in preventing obesity and improving eating habits. However, the study by Sawada et al. [77] expressed the need to carry out interventions that focus exclusively on financial incentives versus no intervention to determine if this strategy has a clear impact. Combined actions could mask these results. 
In line with what was stated by Peñalvo et al. [78], it is important to highlight the generally short/moderate duration (between 6 months and 1 year) of the vast majority of workplace health interventions and programs, as well as the limited evaluation of the sustainability of the change in habits after the end of the program, which may raise doubts about the long-term effectiveness of these interventions. However, in relation to the failure of interventions focused on overweight and obese patients carried out in the workplace, Park and King [72] argue that there is evidence indicating that the duration of the intervention is a determining factor, with short-term programs (less than 6 months) being more effective than long-term programs.

Most of the identified studies came from high-income countries, mainly the United States, where the problem of obesity and overweight has become a heavy burden in economic and health terms for the state and companies [7]. In this sense, and as indicated by Peñalvo et al. [78], occupational health programs and their evaluation are scarce in other geographic and socioeconomic contexts (a single study from India included in the review) where non-communicable diseases are increasing rapidly.

In short, given the substantial period of time that adults spend in their workplaces each day, workplaces provide an opportune environment for interventions relating to healthy habits and can be effective if such interventions combine several strategies (diet, lifestyle, physical activity, reduction in alcohol and tobacco consumption, rewards, adherence to the intervention, etc.) The identification of strategies that are effective in improving the implementation of interventions in the workplace has the potential to improve health outcomes.

However, from the results observed in the clinical trials reviewed, employees acquired a greater awareness of the relationship between diet and health. Additionally, they considered the actions taken a positive experience for themselves and the company. These statements had already been noted in a previous study by Munar-Gelabert et al. [69].

The results of the meta-regression and the little-observed effect derived from the interventions are in line with other previous works. The findings of LaCaille et al. [79], showed that ecological approaches in the workplace have had little or no effect on preventing weight gain. Similarly, Allan et al. [80], in a 2017 systematic review, noted that there was no convincing evidence that this type of intervention resulted in weight or BMI changes. Another limitation of environmental interventions is the cost and levels of administrative approval necessary for modifying the work environment since they can pose a barrier to the implementation and success of environmental strategies. In addition, there may be reluctance regarding healthy alternatives available in the cafeteria and portion size reduction among workers [79]. Moreover, Vermeer et al. [81], noted the importance of assessing the existence of workers' compensatory eating behaviors after eating less in the workplace.

\subsection{Limitations of the Review}

The results of this review are limited by the shortcomings of each work included in it. The level of evidence and recommendation values reached did not ensure that the clinical trials reviewed did not have a high risk of bias. Numerous studies did not specify whether they controlled for confounding factors that could affect the results.

In addition, to raise the level of evidence and recommendation of this review, it would be necessary for all the trials to have taken into account the existence of adverse consequences, an item not observed in any of the included studies. Thus, the low-certainty evidence suggests that such strategies can make little or no difference in the measures of the consistency of implementation or in the different health behavior outcomes of the employees, a circumstance already reported by Wolfenden et al. [76].

\subsection{Critical Analysis of the Authors}

While the majority of clinical trials found that the different interventions observed provided opportunities to establish different programs in the workplace, other studies contradicted this possibility by not finding an association between the intervention group 
and the control group. Additionally, and without doubting the favorable results obtained, many of the trials did not report effects since the intervention ended.

It would have been desirable to have considered the impact that shift work had when implementing the different interventions. This issue was not clear in the documents reviewed.

Another issue that was missed was the absence of information on adherence to the different interventions. As stated by Abbate et al. [82], the follow-up of any strategy is fundamental because it is directly related to health outcomes.

From the meta-regression study, it was observed that the interventions give better results in people who presented high BMI values (obesity). In contrast, intervention 2 (interventions related to workplace environment) would not give the expected results (it would increase the BMI). In addition, although the characteristics of the workplace can generate an obesogenic environment, changes in this environment may be necessary but not enough to modify the obesity-related health behaviors of workers.

Importantly, methodologically rigorous studies are considered necessary to carry out adequate nutritional interventions in the workplace.

\section{Conclusions}

Given that most people spend a large part of their time in the workplace and, therefore, eat at least one of their daily meals there, well-planned interventions-preferably including several strategies-have been shown to be useful for reducing weight, improving healthy behaviors and preventing overweight and obesity.

Author Contributions: Conceptualization, L.M.-F., C.W.-B. and J.S.-V.; methodology, L.M.-F., M.S.-L., P.C. and J.S.-V.; validation, C.W.-B. and J.S.-V.; formal analysis, Á.F.-P., P.C. and M.S.-L.; data curation, L.M.-F., Á.F.-P., P.C. and M.S.-L.; writing-original draft preparation, L.M.-F., C.W.-B. and J.S.-V.; writing-review and editing, L.M.-F., Á.F.-P., M.S.-L., P.C., C.W.-B. and J.S.-V.; supervision, C.W.-B. and J.S.-V. All authors have read and agreed to the published version of the manuscript.

Funding: The authors received no financial support for the research, authorship, and/or publication of this article. The protocol of this systematic review has not been registered.

Institutional Review Board Statement: Not applicable.

Informed Consent Statement: Not applicable.

Conflicts of Interest: The authors declare no conflict of interest.

\section{References}

1. Shearer, J.; Graham, T.E.; Skinner, T.L. Nutra-Ergonomics: Influence of Nutrition on Physical Employment Standards and the Health of Workers. Appl. Physiol. Nutr. Metab. 2016, 41, S165-S174. [CrossRef] [PubMed]

2. Angeles-Agdeppa, I.; Custodio, M.R.S. Food Sources and Nutrient Intakes of Filipino Working Adults. Nutrients 2020, 12, 1009. [CrossRef] [PubMed]

3. Anderson, L.M.; Quinn, T.A.; Glanz, K.; Ramirez, G.; Kahwati, L.C.; Johnson, D.B.; Buchanan, L.R.; Archer, W.R.; Chattopadhyay, S.; Kalra, G.P.; et al. The Effectiveness of Worksite Nutrition and Physical Activity Interventions for Controlling Employee Overweight and Obesity: A Systematic Review. Am. J. Prev. Med. 2009, 37, 340-357. [CrossRef]

4. Melián-Fleitas, L.; Franco-Pérez, Á.M.; Sanz-Valero, J. Bibliometric and Thematic Analysis of Scientific Production on Occupational Health Related to Nutrition, Food and Diet, Indexed in MEDLINE. Med. Segur. Trab. 2019, 65, 10-23. [CrossRef]

5. World Health Organization. Obesity and Overweight. Available online: https://bit.ly/3gIGYwb (accessed on 20 August 2021).

6. Wright, S.M.; Aronne, L.J. Causes of Obesity. Abdom. Imaging 2012, 37, 730-732. [CrossRef]

7. Organisation for Economic Co-Operation and Development (OECD) The Heavy Burden of Obesity: The Economics of Prevention; OECD Health Policy Studies; OECD: Paris, France, 2019; ISBN 978-92-64-33004-7.

8. Engin, A. The Definition and Prevalence of Obesity and Metabolic Syndrome. Adv. Exp. Med. Biol. 2017, 960, 1-17. [CrossRef] [PubMed]

9. Goettler, A.; Grosse, A.; Sonntag, D. Productivity Loss Due to Overweight and Obesity: A Systematic Review of Indirect Costs. BMJ Open 2017, 7, e014632. [CrossRef] [PubMed]

10. Melián-Fleitas, L. Occupational Health New Paradigm: Food, Nutrition and Diet. Med. Segur. Trab. 2019, 65, 73-75. [CrossRef]

11. Lang, J.; Cluff, L.; Payne, J.; Matson-Koffman, D.; Hampton, J. The Centers for Disease Control and Prevention: Findings From The National Healthy Worksite Program. J. Occup. Environ. Med. 2017, 59, 631-641. [CrossRef] [PubMed] 
12. Pelletier, K.R. A Review and Analysis of the Clinical and Cost-Effectiveness Studies of Comprehensive Health Promotion and Disease Management Programs at the Worksite: Update VIII 2008 to 2010. J. Occup. Environ. Med. 2011, 53, 1310-1331. [CrossRef]

13. Piepoli, M.F.; Hoes, A.W.; Agewall, S.; Albus, C.; Brotons, C.; Catapano, A.L.; Cooney, M.-T.; Corrà, U.; Cosyns, B.; Deaton, C.; et al. 2016 European Guidelines on Cardiovascular Disease Prevention in Clinical Practice: The Sixth Joint Task Force of the European Society of Cardiology and Other Societies on Cardiovascular Disease Prevention in Clinical Practice (Constituted by Representatives of 10 Societies and by Invited Experts) Developed with the Special Contribution of the European Association for Cardiovascular Prevention \& Rehabilitation (EACPR). Eur. Heart J. 2016, 37, 2315-2381. [CrossRef] [PubMed]

14. Schröer, S.; Haupt, J.; Pieper, C. Evidence-Based Lifestyle Interventions in the Workplace-An Overview. Occup. Med. Oxf. Engl. 2014, 64, 8-12. [CrossRef] [PubMed]

15. Arksey, H.; O'Malley, L. Scoping Studies: Towards a Methodological Framework. Int. J. Soc. Res. Methodol. 2005, 8, 19-32. [CrossRef]

16. Wanden-Berghe, C.; Sanz-Valero, J. Systematic Reviews in Nutrition: Standardized Methodology. Br. J. Nutr. 2012, 107, S3-S7. [CrossRef]

17. Pandis, N.; Chung, B.; Scherer, R.W.; Elbourne, D.; Altman, D.G. CONSORT 2010 Statement: Extension Checklist for Reporting within Person Randomised Trials. BMJ 2017, 357, j2835. [CrossRef] [PubMed]

18. Harbour, R.; Miller, J. A New System for Grading Recommendations in Evidence Based Guidelines. BMJ 2001, 323, 334-336. [CrossRef]

19. Duval, S.; Tweedie, R. Trim and Fill: A Simple Funnel-Plot-Based Method of Testing and Adjusting for Publication Bias in Meta-Analysis. Biometrics 2000, 56, 455-463. [CrossRef] [PubMed]

20. Copas, J.; Shi, J. A Sensitivity Analysis for Publication Bias in Systematic Reviews. Stat. Methods Med. Res. 2001, 10, 251-265. [CrossRef]

21. Schwarzer, G.; Carpenter, J.R.; Rucker, G. Metasens: Statistical Methods for Sensitivity Analysis in Meta-Analysis. Available online: https: / / cran.r-project.org/package=metasens (accessed on 26 October 2021).

22. Thorndike, A.N.; McCurley, J.L.; Gelsomin, E.D.; Anderson, E.; Chang, Y.; Porneala, B.; Johnson, C.; Rimm, E.B.; Levy, D.E. Automated Behavioral Workplace Intervention to Prevent Weight Gain and Improve Diet: The ChooseWell 365 Randomized Clinical Trial. JAMA Netw. Open 2021, 4, e2112528. [CrossRef] [PubMed]

23. Röhling, M.; Martin, K.; Ellinger, S.; Schreiber, M.; Martin, S.; Kempf, K. Weight Reduction by the Low-Insulin-Method-A Randomized Controlled Trial. Nutrients 2020, 12, 3004. [CrossRef]

24. Iturriaga, T.; Barcelo, O.; Diez-Vega, I.; Cordero, J.; Pulgar, S.; Fernandez-Luna, A.; Perez-Ruiz, M. Effects of a Short Workplace Exercise Program on Body Composition in Women: A Randomized Controlled Trial. Health Care Women Int. 2020, 41, 133-146. [CrossRef]

25. Day, R.S.; Jahnke, S.A.; Haddock, C.K.; Kaipust, C.M.; Jitnarin, N.; Poston, W.S.C. Occupationally Tailored, Web-Based, Nutrition and Physical Activity Program for Firefighters: Cluster Randomized Trial and Weight Outcome. J. Occup. Environ. Med. 2019, 61, 841-848. [CrossRef]

26. Kempf, K.; Röhling, M.; Martin, S.; Schneider, M. Telemedical Coaching for Weight Loss in Overweight Employees: A ThreeArmed Randomised Controlled Trial. BMJ Open 2019, 9, e022242. [CrossRef] [PubMed]

27. Tene, L.; Shelef, I.; Schwarzfuchs, D.; Gepner, Y.; Yaskolka Meir, A.; Tsaban, G.; Zelicha, H.; Bilitzky, A.; Komy, O.; Cohen, N.; et al. The Effect of Long-Term Weight-Loss Intervention Strategies on the Dynamics of Pancreatic-Fat and Morphology: An MRI RCT Study. Clin. Nutr. ESPEN 2018, 24, 82-89. [CrossRef]

28. Viester, L.; Verhagen, E.A.L.M.; Bongers, P.M.; van der Beek, A.J. Effectiveness of a Worksite Intervention for Male Construction Workers on Dietary and Physical Activity Behaviors, Body Mass Index, and Health Outcomes: Results of a Randomized Controlled Trial. Am. J. Health Promot. 2018, 32, 795-805. [CrossRef]

29. Shrivastava, U.; Fatma, M.; Mohan, S.; Singh, P.; Misra, A. Randomized Control Trial for Reduction of Body Weight, Body Fat Patterning, and Cardiometabolic Risk Factors in Overweight Worksite Employees in Delhi, India. J. Diabetes Res. 2017, 2017, 7254174. [CrossRef]

30. Gepner, Y.; Shelef, I.; Schwarzfuchs, D.; Zelicha, H.; Tene, L.; Yaskolka Meir, A.; Tsaban, G.; Cohen, N.; Bril, N.; Rein, M.; et al. Effect of Distinct Lifestyle Interventions on Mobilization of Fat Storage Pools: CENTRAL Magnetic Resonance Imaging Randomized Controlled Trial. Circulation 2018, 137, 1143-1157. [CrossRef]

31. Faghri, P.D.; Simon, J.; Huedo-Medina, T.; Gorin, A. Perceived Self-Efficacy and Financial Incentives: Factors Affecting Health Behaviors and Weight Loss in a Workplace Weight Loss Intervention. J. Occup. Environ. Med. 2017, 59, 453-460. [CrossRef]

32. Geaney, F.; Kelly, C.; Di Marrazzo, J.S.; Harrington, J.M.; Fitzgerald, A.P.; Greiner, B.A.; Perry, I.J. The Effect of Complex Workplace Dietary Interventions on Employees' Dietary Intakes, Nutrition Knowledge and Health Status: A Cluster Controlled Trial. Prev. Med. 2016, 89, 76-83. [CrossRef]

33. Solenhill, M.; Grotta, A.; Pasquali, E.; Bakkman, L.; Bellocco, R.; Trolle Lagerros, Y. The Effect of Tailored Web-Based Feedback and Optional Telephone Coaching on Health Improvements: A Randomized Intervention Among Employees in the Transport Service Industry. J. Med. Internet Res. 2016, 18, e158. [CrossRef] [PubMed]

34. Mitchell, D.C.; Andrews, T.; Schenker, M.B. Pasos Saludables: A Pilot Randomized Intervention Study to Reduce Obesity in an Immigrant Farmworker Population. J. Occup. Environ. Med. 2015, 57, 1039-1046. [CrossRef] 
35. Fernandez, I.D.; Chin, N.P.; Devine, C.M.; Dozier, A.M.; Martina, C.A.; McIntosh, S.; Thevenet-Morrison, K.; Yang, H. Images of a Healthy Worksite: A Group-Randomized Trial for Worksite Weight Gain Prevention With Employee Participation in Intervention Design. Am. J. Public Health 2015, 105, 2167-2174. [CrossRef] [PubMed]

36. Almeida, F.A.; You, W.; Harden, S.M.; Blackman, K.C.A.; Davy, B.M.; Glasgow, R.E.; Hill, J.L.; Linnan, L.A.; Wall, S.S.; Yenerall, J.; et al. Effectiveness of a Worksite-Based Weight Loss Randomized Controlled Trial: The Worksite Study. Obesity 2015, 23, 737-745. [CrossRef]

37. Østbye, T.; Stroo, M.; Brouwer, R.J.N.; Peterson, B.L.; Eisenstein, E.L.; Fuemmeler, B.F.; Joyner, J.; Gulley, L.; Dement, J.M. Steps to Health Employee Weight Management Randomized Control Trial: Short-Term Follow-up Results. J. Occup. Environ. Med. 2015, 57, 188-195. [CrossRef]

38. Van Berkel, J.; Boot, C.R.L.; Proper, K.I.; Bongers, P.M.; van der Beek, A.J. Effectiveness of a Worksite Mindfulness-Based Multi-Component Intervention on Lifestyle Behaviors. Int. J. Behav. Nutr. Phys. Act. 2014, 11, 9. [CrossRef]

39. Mishra, S.; Xu, J.; Agarwal, U.; Gonzales, J.; Levin, S.; Barnard, N.D. A Multicenter Randomized Controlled Trial of a Plant-Based Nutrition Program to Reduce Body Weight and Cardiovascular Risk in the Corporate Setting: The GEICO Study. Eur. J. Clin. Nutr. 2013, 67, 718-724. [CrossRef] [PubMed]

40. Salinardi, T.C.; Batra, P.; Roberts, S.B.; Urban, L.E.; Robinson, L.M.; Pittas, A.G.; Lichtenstein, A.H.; Deckersbach, T.; Saltzman, E.; Das, S.K. Lifestyle Intervention Reduces Body Weight and Improves Cardiometabolic Risk Factors in Worksites. Am. J. Clin. Nutr. 2013, 97, 667-676. [CrossRef]

41. Christensen, J.R.; Overgaard, K.; Carneiro, I.G.; Holtermann, A.; Søgaard, K. Weight Loss among Female Health Care Workers-a 1-Year Workplace Based Randomized Controlled Trial in the FINALE-Health Study. BMC Public Health 2012, 12, 625. [CrossRef]

42. Thorndike, A.N.; Sonnenberg, L.; Healey, E.; Myint-U, K.; Kvedar, J.C.; Regan, S. Prevention of Weight Gain Following a Worksite Nutrition and Exercise Program: A Randomized Controlled Trial. Am. J. Prev. Med. 2012, 43, 27-33. [CrossRef]

43. Linde, J.A.; Nygaard, K.E.; MacLehose, R.F.; Mitchell, N.R.; Harnack, L.J.; Cousins, J.M.; Graham, D.J.; Jeffery, R.W. HealthWorks: Results of a Multi-Component Group-Randomized Worksite Environmental Intervention Trial for Weight Gain Prevention. Int. J. Behav. Nutr. Phys. Act. 2012, 9, 14. [CrossRef]

44. Nanri, A.; Tomita, K.; Matsushita, Y.; Ichikawa, F.; Yamamoto, M.; Nagafuchi, Y.; Kakumoto, Y.; Mizoue, T. Effect of Six Months Lifestyle Intervention in Japanese Men with Metabolic Syndrome: Randomized Controlled Trial. J. Occup. Health 2012, 54, 215-222. [CrossRef]

45. Brehm, B.J.; Gates, D.M.; Singler, M.; Succop, P.A.; D’Alessio, D.A. Environmental Changes to Control Obesity: A Randomized Controlled Trial in Manufacturing Companies. Am. J. Health Promot. 2011, 25, 334-340. [CrossRef]

46. Christensen, J.R.; Faber, A.; Ekner, D.; Overgaard, K.; Holtermann, A.; Søgaard, K. Diet, Physical Exercise and Cognitive Behavioral Training as a Combined Workplace Based Intervention to Reduce Body Weight and Increase Physical Capacity in Health Care Workers-A Randomized Controlled Trial. BMC Public Health 2011, 11, 671. [CrossRef]

47. Barham, K.; West, S.; Trief, P.; Morrow, C.; Wade, M.; Weinstock, R.S. Diabetes Prevention and Control in the Workplace: A Pilot Project for County Employees. J. Public Health Manag. Pract. 2011, 17, 233-241. [CrossRef]

48. Ferdowsian, H.R.; Barnard, N.D.; Hoover, V.J.; Katcher, H.I.; Levin, S.M.; Green, A.A.; Cohen, J.L. A Multicomponent Intervention Reduces Body Weight and Cardiovascular Risk at a GEICO Corporate Site. Am. J. Health Promot. 2010, 24, 384-387. [CrossRef]

49. Maruyama, C.; Kimura, M.; Okumura, H.; Hayashi, K.; Arao, T. Effect of a Worksite-Based Intervention Program on Metabolic Parameters in Middle-Aged Male White-Collar Workers: A Randomized Controlled Trial. Prev. Med. 2010, 51, 11-17. [CrossRef]

50. Siegel, J.M.; Prelip, M.L.; Erausquin, J.T.; Kim, S.A. A Worksite Obesity Intervention: Results from a Group-Randomized Trial. Am. J. Public Health 2010, 100, 327-333. [CrossRef]

51. Van Wier, M.F.; Ariëns, G.A.M.; Dekkers, J.C.; Hendriksen, I.J.M.; Smid, T.; van Mechelen, W. Phone and E-Mail Counselling Are Effective for Weight Management in an Overweight Working Population: A Randomized Controlled Trial. BMC Public Health 2009, 9, 6. [CrossRef]

52. Leslie, W.S.; Lean, M.E.J.; Baillie, H.M.; Hankey, C.R. Weight Management: A Comparison of Existing Dietary Approaches in a Work-Site Setting. Int. J. Obes. Relat. Metab. Disord. J. Int. Assoc. Study Obes. 2002, 26, 1469-1475. [CrossRef] [PubMed]

53. Pritchard, J.E.; Nowson, C.A.; Wark, J.D. A Worksite Program for Overweight Middle-Aged Men Achieves Lesser Weight Loss with Exercise than with Dietary Change. J. Am. Diet. Assoc. 1997, 97, 37-42. [CrossRef]

54. Baer, J.T. Improved Plasma Cholesterol Levels in Men after a Nutrition Education Program at the Worksite. J. Am. Diet. Assoc. 1993, 93, 658-663. [CrossRef]

55. Follick, M.J.; Fowler, J.L.; Brown, R.A. Attrition in Worksite Weight-Loss Interventions: The Effects of an Incentive Procedure. J. Consult. Clin. Psychol. 1984, 52, 139-140. [CrossRef] [PubMed]

56. Hagger, M.S. What Makes a 'Good' Review Article? Some Reflections and Recommendations. Health Psychol. Rev. 2012, 6, 141-146. [CrossRef]

57. World Health Organization. Global Strategy on Occupational Health for All: The Way to Health at Work. Available online: https:/ / bit.ly/2Wt10Gi (accessed on 20 August 2021).

58. Gu, J.K.; Charles, L.E.; Bang, K.M.; Ma, C.C.; Andrew, M.E.; Violanti, J.M.; Burchfiel, C.M. Prevalence of Obesity by Occupation among US Workers: The National Health Interview Survey 2004-2011. J. Occup. Environ. Med. 2014, 56, 516-528. [CrossRef] [PubMed] 
59. Gu, J.K.; Charles, L.E.; Fekedulegn, D.; Ma, C.C.; Andrew, M.E.; Burchfiel, C.M. Prevalence of Injury in Occupation and Industry: Role of Obesity in the National Health Interview Survey 2004 to 2013. J. Occup. Environ. Med. 2016, 58, 335-343. [CrossRef]

60. Muñoz-Cobo-Orosa, B.; Varela-Serrano, C.; Rodriguez-Ledott, M.; Sanz-Valero, J. Malignant Skin Neoplasms in Workers in the Fishing Industry: Systematic Review. Arch. Prev. Riesgos Labor. 2021, 24, 47-61. [CrossRef]

61. Barriocanal-Gómez, P.; Del Pozo-Díez, C.M.; Kudryavtseva, O.; Portillo Chicano, I.; Sanz-Valero, J. Effects Derived from Occupational Exposure to Hazardous Substances in Pregnant Working Women: Systematic Review. Arch. Prev. Riesgos Labor. 2021, 24, 263-296. [CrossRef]

62. Gea Cabrera, A.; Sanz-Lorente, M.; Sanz-Valero, J.; López-Pintor, E. Compliance and Adherence to Enteral Nutrition Treatment in Adults: A Systematic Review. Nutrients 2019, 11, 2627. [CrossRef] [PubMed]

63. Comeche, J.M.; Gutierrez-Hervás, A.; Tuells, J.; Altavilla, C.; Caballero, P. Predefined Diets in Patients with Inflammatory Bowel Disease: Systematic Review and Meta-Analysis. Nutrients 2020, 13, 52. [CrossRef] [PubMed]

64. Begg, C.; Cho, M.; Eastwood, S.; Horton, R.; Moher, D.; Olkin, I.; Pitkin, R.; Rennie, D.; Schulz, K.F.; Simel, D.; et al. Improving the Quality of Reporting of Randomized Controlled Trials. The CONSORT Statement. JAMA 1996, 276, 637-639. [CrossRef] [PubMed]

65. González-Castro, U. Cómo mejorar la calidad de la publicación de ensayos clínicos: La declaración CONSORT. Actas Dermosifiliogr. 2002, 93, 141-142. [CrossRef]

66. Manterola, C.; Asenjo-Lobos, C.; Otzen, T. Hierarchy of Evidence: Levels of Evidence and Grades of Recommendation from Current Use. Rev. Chil. Infectol. 2014, 31, 705-718. [CrossRef]

67. Teufer, B.; Ebenberger, A.; Affengruber, L.; Kien, C.; Klerings, I.; Szelag, M.; Grillich, L.; Griebler, U. Evidence-Based Occupational Health and Safety Interventions: A Comprehensive Overview of Reviews. BMJ Open 2019, 9, e032528. [CrossRef] [PubMed]

68. Cox, N.H.; Williams, H.C. Can You COPE with CONSORT? Br. J. Dermatol. 2000, 142, 1-3. [CrossRef]

69. Munar-Gelabert, M.; Puzo-Foncillas, J.; Sanclemente, T. Dietary-Nutritional Intervention Program for Health Promotion in the Workplace in a Company of the City of Huesca, Spain. Rev. Esp. Nutr. Hum. Diet. 2015, 19, 189. [CrossRef]

70. Álvarez Velásquez, S.; Sanz Valero, J. Ventajas de La Quimioterapia Domiciliaria En Los Enfermos Adultos Con Neoplasias: Revisión Sistemática. Hosp. Domic. 2020, 4, 25-41. [CrossRef]

71. Upadhyaya, M.; Sharma, S.; Pompeii, L.A.; Sianez, M.; Morgan, R.O. Obesity Prevention Worksite Wellness Interventions for Health Care Workers: A Narrative Review. Workplace Health Saf. 2020, 68, 32-49. [CrossRef] [PubMed]

72. Park, S.-H.; Kim, S.-Y. Effectiveness of Worksite-Based Dietary Interventions on Employees' Obesity: A Systematic Review and Meta-Analysis. Nutr. Res. Pract. 2019, 13, 399-409. [CrossRef]

73. Schliemann, D.; Woodside, J.V. The Effectiveness of Dietary Workplace Interventions: A Systematic Review of Systematic Reviews. Public Health Nutr. 2019, 22, 942-955. [CrossRef] [PubMed]

74. Fitzgerald, S.; Geaney, F.; Kelly, C.; McHugh, S.; Perry, I.J. Barriers to and Facilitators of Implementing Complex Workplace Dietary Interventions: Process Evaluation Results of a Cluster Controlled Trial. BMC Health Serv. Res. 2016, 16, 139. [CrossRef]

75. Chu, A.H.Y.; Ng, S.H.X.; Tan, C.S.; Win, A.M.; Koh, D.; Müller-Riemenschneider, F. A Systematic Review and Meta-Analysis of Workplace Intervention Strategies to Reduce Sedentary Time in White-Collar Workers. Obes. Rev. Off. J. Int. Assoc. Study Obes. 2016, 17, 467-481. [CrossRef]

76. Wolfenden, L.; Goldman, S.; Stacey, F.G.; Grady, A.; Kingsland, M.; Williams, C.M.; Wiggers, J.; Milat, A.; Rissel, C.; Bauman, A.; et al. Strategies to Improve the Implementation of Workplace-Based Policies or Practices Targeting Tobacco, Alcohol, Diet, Physical Activity and Obesity. Cochrane Database Syst. Rev. 2018, 11, CD012439. [CrossRef] [PubMed]

77. Sawada, K.; Wada, K.; Shahrook, S.; Ota, E.; Takemi, Y.; Mori, R. Social Marketing Including Financial Incentive Programs at Worksite Cafeterias for Preventing Obesity: A Systematic Review. Syst. Rev. 2019, 8, 66. [CrossRef]

78. Peñalvo, J.L.; Sagastume, D.; Mertens, E.; Uzhova, I.; Smith, J.; Wu, J.H.Y.; Bishop, E.; Onopa, J.; Shi, P.; Micha, R.; et al. Effectiveness of Workplace Wellness Programmes for Dietary Habits, Overweight, and Cardiometabolic Health: A Systematic Review and Meta-Analysis. Lancet Public Health 2021, 6, e648-e660. [CrossRef]

79. LaCaille, L.J.; Schultz, J.F.; Goei, R.; LaCaille, R.A.; Dauner, K.N.; de Souza, R.; Nowak, A.V.; Regal, R. Go!: Results from a Quasi-Experimental Obesity Prevention Trial with Hospital Employees. BMC Public Health 2016, 16, 171. [CrossRef] [PubMed]

80. Allan, J.; Querstret, D.; Banas, K.; de Bruin, M. Environmental Interventions for Altering Eating Behaviours of Employees in the Workplace: A Systematic Review: Environmental Interventions in Eating. Obes. Rev. 2017, 18, 214-226. [CrossRef] [PubMed]

81. Vermeer, W.M.; Steenhuis, I.H.M.; Leeuwis, F.H.; Heymans, M.W.; Seidell, J.C. Small Portion Sizes in Worksite Cafeterias: Do They Help Consumers to Reduce Their Food Intake? Int. J. Obes. 2011, 35, 1200-1207. [CrossRef] [PubMed]

82. Abbate, M.; Gallardo-Alfaro, L.; Bibiloni, M.D.M.; Tur, J.A. Efficacy of Dietary Intervention or in Combination with Exercise on Primary Prevention of Cardiovascular Disease: A Systematic Review. Nutr. Metab. Cardiovasc. Dis. 2020, 30, 1080-1093. [CrossRef] 